\title{
Motion Blur Filtering: A Statistical Approach for Extracting Confinement Forces and Diffusivity from a Single Blurred Trajectory
}

\author{
Christopher P. Calderon* \\ Ursa Analytics, Inc. \\ (Dated: September 5, 2018)
}

\begin{abstract}
Single Particle Tracking (SPT) can aid in understanding a variety of complex spatio-temporal processes. However, quantifying diffusivity and confinement forces from individual live cell trajectories is complicated by inter- \& intra-trajectory kinetic heterogeneity, thermal fluctuations, and (experimentally resolvable) statistical temporal dependence inherent to the underlying molecule's time correlated confined dynamics experienced in the cell. The problem is further complicated by experimental artifacts such as localization uncertainty and motion blur. The latter is caused by the tagged molecule emitting photons at different spatial positions during the exposure time of a single frame. The aforementioned experimental artifacts induce spurious time correlations in measured SPT time series that obscure the information of interest (e.g., confinement forces and diffusivity). We develop a new maximum likelihood estimation (MLE) technique that decouples the above noise sources and systematically treats temporal correlation via time series methods. This ultimately permits a reliable algorithm for extracting diffusivity and effective forces in confined or unconfined environments. We illustrate how our approach avoids complications inherent to mean square displacement (MSD) or autocorrelation techniques. Our algorithm modifies the established Kalman filter (which does not handle motion blur artifacts) to provide a likelihood based time series estimation procedure. The result extends Berglund's motion blur model [PRE, 82 (2010)] to handle confined dynamics. The approach can also systematically utilize (possibly time dependent) localization uncertainty estimates afforded by image analysis if available. To our knowledge, this is the first technique explicitly treating confinement and motion blur within a time domain MLE framework that uses an exact likelihood (time domain methods facilitate analyzing non-stationary signals). Our new estimator is demonstrated to be consistent over a wide range of exposure times $(5-100 \mathrm{~ms})$, diffusion coefficients $\left(1 \times 10^{-3}-1 \mu \mathrm{m}^{2} / \mathrm{s}\right)$, and confinement widths $(100 \mathrm{~nm}-2 \mu \mathrm{m})$. We demonstrate that neglecting motion blur or confinement can substantially bias estimation of kinetic parameters of interest to researchers. The technique also permits one to check statistical model assumptions against measured individual trajectories without "ground truth". The ability to reliably and consistently extract motion parameters in trajectories exhibiting confined and/or non-stationary dynamics, without exposure time artifacts corrupting estimates, is expected to aid in directly comparing trajectories obtained from different experiments or imaging modalities. A Python implementation is provided; open-source code will be maintained on GitHub ${ }^{\text {a }}$.
\end{abstract}

PACS numbers: 87.80.Nj, 02.50.Tt, 87.10.Mn

\section{INTRODUCTION}

The number of techniques available to accurately probe molecules in their native, crowded, and time changing live cell environment has increased dramatically in recent years [1-27]. Research aimed at more efficiently extracting kinetic information from live cell single particle tracking (SPT) experiments has also experienced rapid growth [28-42]. However, analysis methods have substantially lagged behind microscopy developments. An important and ubiquitous problem in cell biology [43, 44] that has not received substantial statistical attention is associated with how to address various technical challenges inherent to analyzing confined motion $[17, \underline{45}, \underline{46}]$

\footnotetext{
* Chris.Calderon@UrsaAnalytics.com

a This version differs in minor typographical details from the published version, DOI:10.1103/PhysRevE.93.053303, available on-line at:http://journals.aps.org/pre/abstract/ 10.1103/PhysRevE.93.053303. Code will be maintained at http://github.com/calderoc/MotionBlurFilter.
}

in a collection of experimental trajectories exhibiting heterogeneous and/or non-stationary (i.e., transient kinetic phenomena) responses [33, 41, 44, 47-49]. High-resolution multicolor image stacks can provide hints of molecular interactions when analyzed via a spatial co-localization analysis [27], however reliably distinguishing between transient molecular binding events (hence changing the underlying molecular diffusivity of the biomolecule) vs. coincidental co-localization can be aided by new quantitative time series methods. Measurement apparatus noise further complicate the problem, since in optical microscopy, position measurements are not "instantaneously" observed. Empirically measured data is a time averaged quantity $[29, \underline{46}, 50]$. For example, in fluorescence based optical microscopy $[7,16,51]$, position is inferred from the observed Point Spread Function (PSF) [16, 51-53] obtained by collecting a finite number of photons emitted as the tagged molecule moves throughout the cell $[16,29,38,46,50,54]$. The noise due solely to photon emissions from multiple positions in a single image is what we refer to generically as "motion blur". The statistical correlation between thermal fluctuations, confinement forces, and motion blur 
introduces new time series challenges not addressed by current SPT data analysis routines. Refs. [29, 37] address how to handle motion blur issues under the assumption of simplified diffusion models, but fail to address technical complications associated with confined motion (a common occurrence in live cell SPT studies). The classic Kalman Filter (KF) algorithm can deal with simple confined motion models, but fundamental assumptions behind the KF are violated when motion blur is present $[55,56]$.

We introduce a new likelihood based estimation scheme that explicitly models (i) the spatio-temporal statistical correlation inherent to molecular position measurements undergoing confined diffusion [32, 46]; and (ii) the correlation between various sources of measurement noise and the underlying particle position time series in camera based measurements (the approach treats "dynamic" and "static" measurements errors commonly encountered in SPT [38]). The technique is capable of reliably estimating local molecular diffusivity, $D$, and instantaneous velocity $\&$ forces from a single noisily measured position vs. time SPT trajectory. Reliably estimating the aforementioned kinetic quantities from SPT trajectory measurements requires one to address statistical correlation induced by confinement, localization, and motion blur [46]. Obtaining reliable estimates of instantaneous forces from a single trajectory and respecting heterogeneity commonly encountered in SPT data [33, $11, \underline{44}, \underline{47}-49]$ requires an accurate estimate of $D$ that is free of measurement apparatus artifacts (our approaches provides this information). Our approach also enables researchers to use a single measured trajectory produced by an individual molecule (where localization quality may vary over time) to systematically decouple "dynamic measurement errors" from effective "static measurement errors" [으, 46, 50]. This decomposition is described in detail later since it is a key technical aspect of our new algorithm.

As we demonstrate, failing to properly account for motion blur and/or confinement can substantially affect quantities required to estimate forces from SPT trajectories. The Motion Blur Filter (MBF) algorithm addresses the technical concerns through a reformulation of the $\mathrm{KF}$ $[55,56]$. To our knowledge, this is the first time domain likelihood based approach that explicitly accounts for confinement and motion blur. Likelihood-based approaches accounting for the natural time ordering of measurements $[33,57,58]$ are advantageous since transient (but experimentally resolvable) changes in molecular forces cannot be readily detected by legacy approaches such as mean square displacement (MSD) [46] or autocorrelation approaches $[38,50]$ where implicit assumptions about stationary statistics are made. The MBF algorithm utilizes closed-form analytical expressions from stochastic process theory and avoids ad hoc statistical approximations to the likelihood function. The ability of our technique to consistently estimate diffusion coefficients ranging from $1 \times 10^{-3}$ to $1 \mathrm{\mu m}^{2} / \mathrm{s}$ in the presence of molecular confinement sampled using observations spaced "finely" (5 $m s)$ to "coarsely" (100 ms) in time is demonstrated via simulations.

Our approach does assume that some approximate localization technique [16, 51-53, 59], e.g. centroid or PSF shape based, can extract an unbiased estimate of the time averaged position (where averaging occurs over the exposure time of a single image) of the molecule of interest; note that the "quality "of the localization is permitted to vary over time within our framework. A data-driven technique, processing "blurred" data, capable of decoupling various noise sources over a wide range of exposure times is expected to aid in identifying physically relevant motion experienced in vivo in a variety of dynamic processes. The MBF provides an algorithm enabling a more direct and reliable comparison of parameters obtained from data with different temporal and spatial resolution since biases affecting other methods at different length and time scales are not experienced by the MBF. This is expected to result in a more comprehensive picture of dynamics occurring in the cell.

Note that when biomolecules are sampled with singlemolecule precision in vivo, they often experience transitions from "standard diffusion" to "anomalous diffusion" as the timescale of observation increases [43]. A longer term aim of this work is to provide a statistically robust method capable of accurately quantifying the motion parameters associated with single-molecule data before events leading to "anomalous diffusion" phenomena manifest and can be statistically detected within individual trajectories. Our motivation is to extract "finer scale" molecular kinetic information from the high temporal and spatial resolution measurements afforded by contemporary optical microscopy with the hope of providing a tool which can accelerate detection of new dynamic phenomena from these measurements [1- $1, \underline{4}-27]$.

This article is organized as follows: Section II introduces the models, theoretical background, and the new MBF algorithm. Towards the end of this section (in subsection IIF), we walk the reader through two examples illustrating how to use the open-source Python code and IPython Notebooks provided along with this article (these examples reproduce Figs. 3 and 4). Section III presents

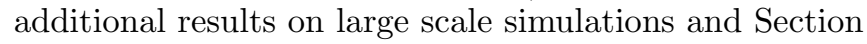
IV concludes. An Appendix provides additional mathematical details. The Supp. Mat. [60] provides additional results and algorithmic details.

\section{MODEL AND METHODS}

This subsection is organized as follows: the assumed continuous time dynamical model is introduced in Sec. II A. Prior to discussing the finer technical time series and filtering details, we expand on the assumptions made about the measurement noise sources in Sec. II B, since these assumptions are key to understanding the MBF. After a detailed description of the measurement noise, we briefly discuss physical interpretations of the model 
in Sec. IIC (additional details can be found in Refs. [33] and 41]). This discussion is followed by a review of the classic KF in Sec. II D. After providing the required background, in Sec. II E we introduce the MBF algorithm. Subsequently, we provide two illustrative examples in Sec. IIF illustrating advantages of the MBF and how one can leverage the approach on SPT data. This section concludes by contrasting the MBF to existing approaches in SPT.

\section{A. Continuous Time Model with Discrete "Blurred" Measurements}

The MBF assumes the following Stochastic Differential Equation (SDE) and measurement model:

$$
\begin{aligned}
& d r_{t}=\left(v-\kappa r_{t}\right) d t+\sqrt{2 D} d B_{t} \\
& \psi_{t_{i}}=\frac{1}{t_{E}} \int_{t_{i}-t_{E}}^{t_{i}} r_{s} d s+\epsilon_{t_{i}}^{\mathrm{loc}}
\end{aligned}
$$

The true position of the tagged particle at time $t$ is denoted by $r_{t}$ [61] and the discretely sampled position measured by the microscope is denoted by $\psi_{t_{i}}$. The stochastic term driving the SDE above is a standard Brownian motion, denoted by $B_{t}$. The effective "static" $[38,52-54,59]$ localization measurement noise at $t_{i}$ measured by a camera with exposure time $t_{E}$ is denoted by $\epsilon_{t_{i}}^{\text {loc }}$ (this noise is modeled as a mean zero Gaussian random variable independent of the particle position). The time integral in Eq. 2 models "motion blur" introduced by the camera [16, 29] [62]. Additional assumptions and details regarding the measurement noise sources are discussed later in this section and in greater detail in Sec. II B. The model above is specified by a parameter vector denoted by $\theta=\left(v, \kappa, D, \sigma_{\text {loc }}\right)$ (this parameter vector contains physically interpretable parameters described in Secs. II B - II C).

The continuous time SDE in Eq. 1 is a linear model whose solution is known precisely $[63, \overline{6} 4]$. Using $\kappa>0$ allows confinement to be modeled (a common phenomenon in live cell studies). However, the spatial and temporal dependence introduced when $\kappa \neq 0$ and motion blur corrupts observations poses several new time series challenges not addressed previously [29, 33]. The MBF algorithm processes discrete measurements samples taken from the continuous time SDE. The linear and Gaussian nature of Eq. 1 enables an exact discretization of Eq. 1, as well as a discrete representation of the integral appearing in Eq. 2 that does not introduce any statistical errors into the filtering framework (these features are crucial to the MBF). The aforementioned discretization of Eqs. 1-2 is given by:

$$
\begin{aligned}
& r_{t_{i+1}}=A+F r_{t_{i}}+\eta_{t_{i}} \\
& \psi_{t_{i}}=H_{A}+H_{F} r_{t_{i-1}}+\epsilon_{t_{i}}^{\text {loc }}+\epsilon_{t_{i}}^{\text {mblur }} \\
& \epsilon_{t_{i}}^{\text {loc }}+\epsilon_{t_{i}}^{\text {mblur }} \sim \mathcal{N}\left(0, R_{i}\right) \\
& \eta_{t_{i}} \sim \mathcal{N}(0, Q) \\
& \epsilon_{t_{i}}^{\text {mblur }} \sim \mathcal{N}\left(0, Q^{\text {mblur }}\right) \\
& C=\operatorname{cov}\left(\eta_{t_{i}}, \epsilon_{t_{i+1}}^{\text {mblur }}\right) \\
& \operatorname{cov}\left(\eta_{t_{i}}, \epsilon_{t_{j}}^{\text {mblur }}\right)=0 \text { if } j \neq i+1 \\
& \operatorname{cov}\left(\eta_{t_{i}}, \epsilon_{t_{j}}^{\text {loc }}\right), \operatorname{cov}\left(\epsilon_{t_{i}}^{\text {loc }}, \epsilon_{t_{j}}^{\text {mblur }}\right)=0 \forall i, j
\end{aligned}
$$

where $\mathcal{N}\left(\mu, \sigma^{2}\right)$ denotes a Gaussian random variable with mean $\mu$ and variance $\sigma^{2}$. The quantities $\left(A, C, F, H_{A}, H_{F}, Q, Q^{\text {mblur }}, R_{i}\right)$ listed in the discrete equations above can be derived in closed-form given $\delta_{i}:=t_{i}-t_{i-1}, t_{E}$, and the continuous time parameters $\theta$ (expressions not explicitly defined in this section are derived and provided in the Appendix). The term $\eta_{t_{i}}$ represents a Gaussian mean zero "process noise" $[55,56]$ with variance $Q$; the term "process noise" is used in control theory to describe a stochastic noise source that affects the true underlying state of the system (the "state" is molecular position in the application considered here). The term $\epsilon_{t_{i}}^{\text {mblur }}$ represents the difference between $r_{t_{i}}$ and the conditional expectation of $\frac{1}{t_{E}} \int_{t_{i}-t_{E}}^{t_{i}} r_{s} d s$ (conditioned on $\left.r_{t_{i-1}}\right)$.

An important technical aspect of the discretized model above is the fact that the conditional expectation $\mathbb{E}\left[\frac{1}{t_{E}} \int_{t_{i}-t_{E}}^{t_{i}} r_{s} d s \mid r_{t_{i-1}}\right]$ is a Gaussian random variable with mean $H_{A}+H_{F} r_{t_{i-1}}$ and variance $Q^{\text {mblur }}$. The parameters $H_{A}$ and $H_{F}$ are used to compute the expected value of the measurement at the next time instant (since there is motion blur, the mean of the measurement does not coincide with the value of the underlying position at the same time point). The parameters $A$ and $F$ play a similar role, i.e., $\mathbb{E}\left[r_{t_{i}} \mid r_{t_{i-1}}\right]=A+F r_{t_{i-1}}$ ( $A$ and $F$ type parameters are commonly encountered in KF applications when a continuous time model is inferred from discretely observed measurements [32]).

Equation 8 emphasizes that $\epsilon_{t_{i+1}}^{\text {mblur }}$ is statistically correlated with the $\eta_{t_{i}}$ (all other noise terms above are statistically independent). The net measurement noise variance obtained by combining the static and dynamic error is $R_{i}:=Q^{\text {mblur }}+\left(\sigma_{\text {loc }}^{\text {Input }}\left(t_{i}\right)+\sigma_{\text {loc }}\right)^{2}$ (the term in parenthesis permits time dependent effective static measurement noise; these terms are described in detail in the next subsection \& Illustrative Example 2 in Sec. IIF). Figure 1 illustrates the motion blur measurement noise and effective static measurement noise sources graphically (these terms are further described in Sec. IIB).

Under the assumed parametric model (see Eqs. 1 and 2 ), the variance of $\epsilon_{t_{i}}^{\text {mblur }}$ and its covariance with $r_{t_{i}}$ (covariance quantified by $C$ in Eq. 8) can be obtained in closed-form in terms of the model parameters (derivation 
provided in Appendix). The classic Kalman Filter (KF) can account for confinement forces and non-stationary statistics [33], but the classic KF is not able to explicitly handle motion blur due to the correlation between $\epsilon_{t_{i}}^{\text {mblur }}$ and $\eta_{t_{i-1}}$. Both the practical utility and main technical contribution of the MBF are associated with how the $M B F$ handles Eq. 2 and the correlation in Eq. $\underline{8}$, hence a detailed qualitative description of the physical phenomena underlying the assumptions behind the "measurement error" terms is presented before proceeding.

\section{B. Qualitative Description of Static and Dynamic Measurement Errors}

In SPT analysis of fluorescence optical microscopy data, the position measurements of a single molecule are typically obtained from through localization techniques [38, 52- 54, 59]. Throughout the remainder of this article, the term "PSF" is used to represent the observed Point Spread Function. The inference of the PSF can be guided by optics principles, but we use the term "PSF" to represent a data-driven quantity extracted from an image measured by an optical light microscope. If the molecule being imaged by a microscope is mobile, the observed PSF is contaminated by both "static" and "dynamic" measurements errors $[29, \underline{38}, \underline{46}, \underline{50]}$. The integral in Eq. 2 models the "dynamic" motion blur measurement error, $\bar{\epsilon}_{t_{i}}^{\text {mblur }}$, due to the tagged molecule emitting photons at different spatial positions in the time interval producing a single image, $\left[t_{i}-t_{E}, t_{i}\right]$. The motion blur measurement error is highly correlated with the underlying particle position of interest. The effective "static" measurement error is intended to quantify the error associated with inferring a PSF's shape from observed pixel intensities [38, 52-54, 59]. That is, the effective static measurement error is an idealized quantity (estimated from observational data) which aims at quantifying the error due to estimating a PSF from a finite number of measured photons. The dynamic or immobile nature of the emitters producing the data used to calibrate the PSF is not relevant to the data-driven effective static measurement noise used by the MBF. Note that the observed PSF may not be radially symmetric even in the "ideal infinite photon" limit due to the dynamic errors introduced by molecular motion. However, as stated in the introduction, the MBF does assume that the localization procedure provides an unbiased point estimate of the the time averaged position of the molecule of interest in the image measurement (the mean zero assumption of $\epsilon^{\text {loc }}$ in Eq. 4 reflects this assumption). In an ideal "infinite photon collection" scenario, the variance of $\epsilon^{\text {loc }}$ would be zero. However, even in the infinite photon case, there would still be uncertainty in the molecular position if the molecule moves and the exposure time is non-zero. Both measurement errors are illustrated in Fig. 1.

The main assumptions underlying the MBF are that (i) the dynamics are consistent with Eqs. 1 and (ii) the error

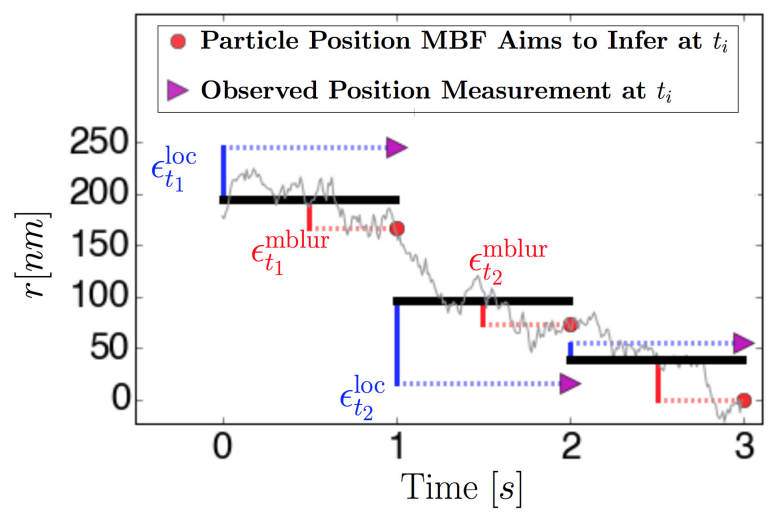

FIG. 1. Illustration of "Dynamic" and Effective "Static" Measurement Errors. The solid gray line represents the true (unobservable) position trajectory of the molecule evolving in continuous time. The trajectory measured without noise contains temporal autocorrelation due to confinement forces [29, 38, 46, 50]. The thick black horizontal lines denote the time averaged position recorded during the camera's exposure time, $t_{E}$. The Motion Blur Filter (MBF) aims to infer the position at time $t_{i}$ (denoted by red circles) from a sequence of noisy measurements, $\left\{\psi_{t_{i}}\right\}_{i=1}^{T}$ (denoted by purple triangles). Each measurement, $\psi_{t_{i}}$, is assumed to come from a Point Spread Function (PSF) generated by a single molecule emitting a finite number of photons at different positions during image acquisition ("image $i$ "is measured during the time interval $\left.\left[t_{i}-t_{E}, t_{i}\right]\right)$. This induces what we refer to as "motion blur" measurement error and this noise is denoted by $\epsilon_{t_{i}}^{\text {mblur }}$. The idealized effective static localization error (induced by finite photon counts in PSF estimation) is denoted by $\epsilon_{t_{i}}^{\text {loc }}$. See text for additional details on the assumptions behind these measurement noise sources.

introduced by finite photon count PSF estimation can be approximated by a Gaussian random variable whose statistics are determined by Eq. 2. The MBF framework recognizes that within empirically observed measurements, static and dynamic measurement noise sources are convolved in the raw data. The MBF approach uses a datadriven model-based approach to "decouple" static and dynamic errors. Although the position measurement is blurred due to molecular motion during image acquisition and finite camera exposure time [29, 38, 46, 50], the MBF aims at rigorously inferring the instantaneous position of the molecule at $t_{i}$ as well as the parameters characterizing its motion. The SDE model-based framework outlined in Eqs. 3 - 10 is key to achieving the noise decomposition described in this section. In most SPT applications, the Gaussian assumption on the effective static measurement noise cannot typically be statistically rejected if 10 or more photons underlie a PSF estimate (formal means for detecting Poisson artifacts could be considered [65]). In this article, goodness-of-fit tests are used to detect if data is consistent with various assumptions underlying the assumed dynamical models (the tests used have been shown capable of detecting artifacts of "low photon count" measurement error in SPT data if they are present in the data [65]).

In the open-source software provided (discussed in Sec. IIF) with this article, we allow estimates of time de- 
pendent localization noise statistics through the optional input $\sigma_{\text {loc }}^{\text {Input }}\left(t_{i}\right)$. The time dependent input estimate of the localization uncertainty at time $t_{i}$ can be biased (e.g., it may contain artifacts of motion blur). When the optional sequence $\sigma_{\mathrm{loc}}^{\mathrm{Input}}\left(t_{i}\right)$ is input, the MBF uses the full sequence of measurements to estimate the parameter $\sigma_{\text {loc }}$ which defines the net effective time dependent static measurement noise variance: $\left(\sigma_{\text {loc }}^{\text {Input }}\left(t_{i}\right)+\sigma_{\text {loc }}\right)^{2}$ for each $i$. It is stressed that the net effective static measurement error is estimated from the data and not assumed known $a$ priori (the parameter $\sigma_{\mathrm{loc}}$ adjusts for the fact that the input localization estimates are likely corrupted by motion blur or other artifacts). If $\sigma_{\text {loc }}^{\text {Input }}\left(t_{i}\right)$ is not provided as input, the estimated parameter $\sigma_{\text {loc }}$ denotes the constant effective static measurement noise associated with the time series data.

\section{Physical Interpretation of Continuous Time SDE Parameters}

Recall that the SDE of interest (Eq. 1) is characterized by the parameter vector $\theta=\left(v, \kappa, D, \sigma_{\text {loc }}\right)$ where $D$ denotes the local effective diffusion coefficient; $\kappa$ and $v$ characterize the instantaneous velocity $[33,35,41]$. The parameter $\sigma_{\text {loc }}$ was described in detail in the previous section. In live cell studies, motion is often confined and confinement affects the temporal correlation statistics [46] even if motion blur and measurement artifacts are not present in the data. The SDE model considered can use a single trajectory to compute "the instantaneous force" from the estimated diffusion coefficient [41]. The ability to use a single trajectory to estimate motion parameters permits researchers to quantify heterogeneity and time changing forces at different points in the cell [41]. For example, the effective force at time $t$, denoted by $F(t)$, is approximated by $\frac{k_{B} T}{\hat{D}}\left(\hat{v}-\hat{\kappa} r_{t}\right)$ where hats denote the maximum likelihood estimate (MLE) extracted from the data. In the previous force equation, we appealed to the classical Einstein relationship, i.e., $D=\frac{k_{B} T}{\gamma}$, where $k_{B} T$ is Boltzmann's constant multiplied by the system temperature and $\gamma$ is the effective molecular friction $[33,63]$. Our technique for estimating forces assumes that "cage hopping" or "crowding" events have not occurred within the observed trajectory $[38, \underline{43}, \underline{48}, \underline{66}]$. Note that we are not restricting the term "cage hopping" to refer to kinetic phenomena in membrane diffusion [45, 46]. In the presence of SPT trajectories spanning "long times", we acknowledge that crowding in the cell may result in cage hopping type phenomena. In Sec. IV, we discuss techniques to preprocess trajectories by segmenting the data into regimes where the MBF technique can be used to extract reliable force from position vs. time data using the model above as a building block. If cage hopping type phenomena occurs on time scales much faster than the temporal resolution afforded by the measurement device (e.g., many "cage jumps" can occur during the exposure time), the MBF can still be used to estimate effective SDE parameters explicitly accounting for the statistical effects of motion blur.

In the remainder of this subsection, we illustrate how the SDE above nests other popular SPT models (i.e., we outline how directed and pure diffusion models are special cases of the SDE model in Eq. 1). If both $v$ and $\kappa$ are set to zero, one obtains the motion blur model considered by Berglund [29] (handling the $v \neq 0, \kappa=0$, is a simple extension of Berglund's result as we show in the code supplied). When $\kappa \neq 0$, one has an Ornstein-Uhlenbeck (OU) process [63]. If $\kappa>0(\kappa \geq 0$ is assumed by the $\mathrm{MBF}$ ), the OU process can model confined diffusion. A popular SPT confinement parameter, $L$ [45], is closely connected to the so-called "corral radius". The original usage of the parameter $L$ in SPT corresponded to a box width (not a proper "radius") of a hard wall potential [45]. However, the "coral radius" term is often used in SPT to generically describe the square root of the asymptotic limit of an MSD curve of a confined particle $[32, \underline{46}, \underline{67}$. $L$ is related to the parameters of the model considered here through the equality $L=\sqrt{\frac{12 D}{\kappa}}$ (see Ref. [32]).

\section{Basics of Kalman Filtering (KF)}

In this subsection, we review the key assumptions underlying the classic $\mathrm{KF}$ at a high level since the MBF makes several modifications to this established algorithm $[55,56]$. The KF assumes that a linear dynamical system can be used to describe the evolution of the "state" (the "state" is the molecular position $r_{t_{i}}$ in our application and is not directly observable due to the measurement noise). The "process noise" (i.e., the Brownian noise in Eq. 3) and net measurement noise are all assumed to be governed by Gaussian statistics in the classic KF. The KF leverages the following mathematical principle. Assume the random vector, $\left[\begin{array}{c}X \\ Y\end{array}\right] \sim \mathcal{N}(\vec{\mu}, \Sigma)$ where the mean, $\vec{\mu}=\left[\begin{array}{c}\mu_{X} \\ \mu_{Y}\end{array}\right]$, and covariance, $\Sigma=\left[\begin{array}{cc}\Sigma_{X X} & \Sigma_{X Y} \\ \Sigma_{X Y} & \Sigma_{Y Y}\end{array}\right]$, specifying the Gaussian are known; $\Sigma_{X X}$ is the variance of $X$ (similarly for $\Sigma_{Y Y}$ ) and $\Sigma_{X Y}:=\operatorname{cov}(X, Y)$ is the covariance of $X$ and $Y$. Assume $Y$ is directly observable, but $X$ is not. In this case, the linear minimum variance estimate of $X$ given $Y$, denoted by $\mathbb{E}^{*}[X \mid Y]$ is given by [56]:

$$
\mathbb{E}^{*}[X \mid Y]=\mu_{X}+\Sigma_{X Y} \Sigma_{Y Y}^{-1}\left(Y-\mu_{Y}\right)
$$

The above relationship is a general principle used by multiple estimators, not just the KF [56]. A useful aspect of the $\mathrm{KF}$ algorithm is associated with the fact that a time series of measurements can be efficiently and sequentially processed building off of the general relationship in Eq. 11. To illustrate the sequential aspect and specialize to notation used in the discretized version of our model (Eqs. $\underline{3}$ and $\underline{4}$ ), we use Fig. $\underline{2}$ and the following equation: 
$\hat{r}_{i+1 \mid i+1}=\hat{r}_{i+1 \mid i}+\operatorname{cov}\left(r_{i+1}, \tilde{\psi}_{i+1}\right) \operatorname{cov}\left(\tilde{\psi}_{i+1}, \tilde{\psi}_{i+1}\right)^{-1} \tilde{\psi}_{i+1}$,

where $\tilde{\psi}_{i+1}:=\psi_{i+1}-\hat{\psi}_{i+1 \mid i}, \hat{\psi}_{i+1 \mid i}$ is the "Forecasted Measurement" (the expected value of $\psi_{i+1}$ ) conditioned on the model parameters and all previously observed measurements up to time $t_{i}$ (the "Forecasted State", $\hat{r}_{i+1 \mid i}$, is analogously defined, but is used to predict $\left.r_{i+1}\right) . \hat{r}_{i+1 \mid i+1}$ represents the "filtered state" estimate (i.e., the linear minimum variance estimate of $r_{i+1}$ given all information available up to time $\left.t_{i+1}\right)$. The sequence $\left\{\tilde{\psi}_{i}\right\}_{i=1}^{T}$ is referred as the "innovation sequence". Eq. 12 is the time sequential KF analog of Eq. 11. The subscript $t$ has been omitted from all quantities to simplify notation when dealing with discrete equations and filter algorithms (observing a subscript $i$ is equivalent to $t_{i}$ ). The various boxes in Fig. 2 compute different quantities in Eq. 12. For example, the "Forecast State" box is one step of the algorithm and provides $\hat{r}_{i+1 \mid i}$. In the classic KF, the "Forecast Measurement" box is an algorithmic step providing $\hat{\psi}_{i+1 \mid i}$ given $\hat{r}_{i+1 \mid i}$ (the corresponding MBF module uses a different input). The "Corrector" step of the algorithm combines the aforementioned forecasts and the actual measurement $\psi_{i+1}$ to produce, $\hat{r}_{i+1 \mid i+1}$. At the top of the diagram, we show that the forecasted measurements and observed measurement can be used to compute a likelihood score. Beyond just providing an estimate of the unobservable position $\hat{r}_{i+1 \mid i+1}$, the KF can provide the MLE. The MLE, $\hat{\theta}$, is obtained by maximizes the sum of $\log$ likelihood scores associated with the observed $\left\{\psi_{i}\right\}_{i=1}^{T}$ over $\theta[55]$.

Traditional KF measurement equations [55, 56] often assume that an a priori known linear transformation, $H$, which maps the state at the target filter time of interest $\left(t_{i+1}\right)$ to the measurement vector is available, i.e. $\psi_{i+1}=$ $H r_{i+1}+$ "measurement noise" and the aforementioned measurement noise does not depend on past values of $r_{s}$ for $s<t_{i+1}[55,56]$. Both conditions are violated for the motion blur model considered here and elsewhere $[29,38,46,50]$. The time integral in Eq. 2 is distributed as a Gaussian, but the mean of this measurement is in terms of $r_{i}$ vs. $r_{i+1}$ for measurement $\psi_{i+1}$. Also, the measurement noise in Eq. 4 is statistically dependent on $r_{s}$ for $s<t_{i+1}$. More advanced treatments of the KF show how to account for "Kronecker delta" type time correlations, i.e. $\delta_{i j}$, between measurement and process noise (e.g., [56]), however the time index offset shown in Eq. 8 causes the KF technical challenges $[55,56]$. The $\mathrm{MBF}$ overcomes these complications by fundamentally changing how $\hat{r}_{i \mid i}$ is processed (see Fig. 2) as we outline in the next subsection.
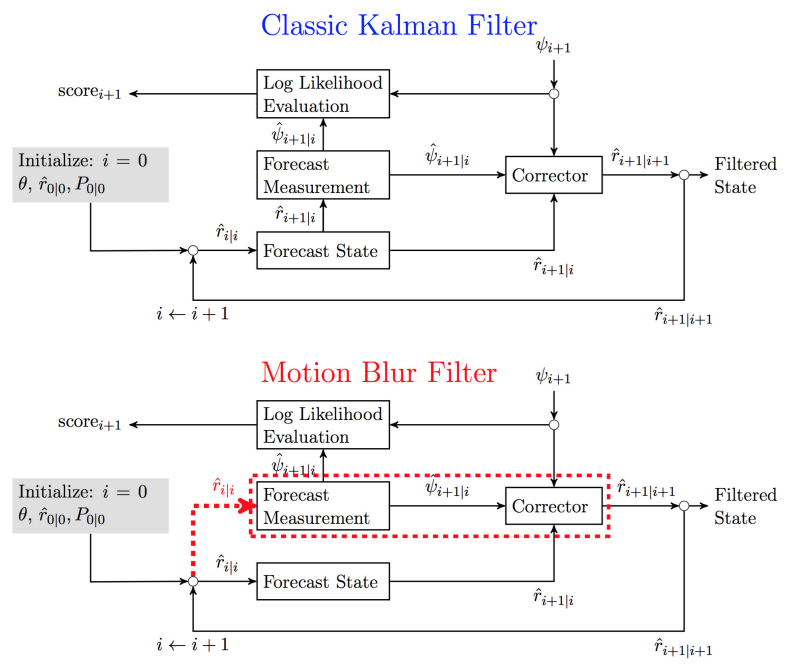

FIG. 2. Graphical Illustration of Kalman Filter (KF) and Motion Blur Filter (MBF). Each filter requires the parameters governing the process $\theta$ as well as the mean $\hat{r}_{0 \mid 0}$ and covariance matrix $P_{0 \mid 0}$ of the initial state as input. Both filters sequentially processes the measurements, $\psi_{i+1}$ to generate $\hat{r}_{i+1 \mid i+1}$ which are estimates of the (unobservable) state $r_{i+1}$ at time $t_{i+1}$. Two byproducts of each filter are: (I) a likelihood score of the observation for the input $\theta$ (the sum of the logarithms of the likelihoods can be used to obtain the maximum likelihood estimate, $\hat{\theta}$ [55]) and (II) summary statistics of both $r_{i+1}$ and $\psi_{i+1}$; means are denoted by $\hat{r}_{i+1 \mid i}, \hat{\psi}_{i+1 \mid i}$, respectively (the covariances are also computed, but omitted from the flow diagram to aid figure clarity). The top panel displays the classic discrete sequential $\mathrm{KF}$ and the bottom shows the new MBF. The key algorithmic differences between the KF and MBF are highlighted by red dashed lines (see Sec. IIE for mathematical details).

\section{E. The MBF Algorithm}

In this section we provide the equations and pseudocode outlining the MBF (recall the underlying discrete model processed by the MBF was presented in Eqs. 3 - 10). The "parallel "processing of $\hat{r}_{i \mid i}$ by the Forecast Measurement and Forecast State modules shown in Fig. 2 vs. the sequential processing used by the classic KF is one key aspect that distinguishes MBF from the KF. The parallel processing of $\hat{r}_{i \mid i}$ is used because the time integral in Eq. 2 violates core assumptions behind the KF $[55,56]$ (discussed in previous section). Since the measurement noise induced by motion blur at $t_{i+1}$ depends on the process noise experienced in the interval $\left[t_{i}, t_{i+1}\right]$, the MBF measurement forecast depends on linear transformations of $\hat{r}_{i \mid i}$ and its covariance (vs. $\hat{r}_{i+1 \mid i}$ and its covariance as in the classic KF).

The parallel processing of $\hat{r}_{i \mid i}$ mentioned above requires some modifications to modules of the classic KF algorithm shown in Fig. 2. The modified "Forecast Measurement" equation reads: 


$$
\begin{aligned}
\hat{\psi}_{i+1 \mid i} & =H_{A}+H_{F} \hat{r}_{i \mid i} \\
S & =H_{F} P_{i \mid i} H_{F}^{\top}+R_{i}
\end{aligned}
$$

and the modified "Corrector" (also known as the "Measurement Update" [56]) equation reads:

$$
\begin{aligned}
K: & =\left(C+F P_{i \mid i} H_{F}^{\top}\right)\left(H_{F} P_{i \mid i} H_{F}^{\top}+R_{i}\right)^{-1} \\
\hat{r}_{i+1 \mid i+1} & =\hat{r}_{i+1 \mid i}+K\left(\psi_{i+1}-\hat{\psi}_{i+1 \mid i}\right) \\
P_{i+1 \mid i+1} & =P_{i+1 \mid i}-K\left(H_{F} P_{i \mid i} H_{F}^{\top}+R_{i}\right) K^{\top}
\end{aligned}
$$

where the filter state forecast $\left(\hat{r}_{i+1 \mid i}\right)$, the filtered state $\left(\hat{r}_{i+1 \mid i+1}\right)$, and the measurement forecast $\left(\hat{\psi}_{i+1 \mid i}\right)$ described in the previous section have covariances $P_{i+1 \mid i}$, $P_{i+1 \mid i+1}$ and $S$, respectively. We use $S$ to denote the "innovation covariance" [56] (the notation $P_{i+1 \mid i}$ and $P_{i+1 \mid i+1}$ is common in the $\mathrm{KF}$ framework $[55,56])$. Recall that the parameters $A, C, F, H_{A}, H_{F}, Q, R_{i}$ are associated with the statistically precise discretized version of Eqs. 1 - 2 and assume $t_{E}=t_{i+1}-t_{i}$ for all $i$, (expressions for these parameters, depending on $t_{E}$, are provided in the previous section and Appendix).

Note that the altered measurement forecasts affects the form of the classic "Corrector" or "Measurement Update" equations [56]. The measurement noise induced by motion blur is correlated to the process noise under the assumed model and this changes the standard form of the "Corrector" update (Eq. 12), specifically under the MBF model:

$$
\begin{array}{r}
\operatorname{cov}\left(r_{i+1}, \psi_{i+1}\right):=\operatorname{cov}\left(r_{t_{i+1}}, \psi_{t_{i+1}}\right) \\
=\operatorname{cov}\left(A+F r_{t_{i}}+\eta_{t_{i}}, H_{A}+H_{F} r_{t_{i}}+\epsilon_{t_{i+1}}^{\text {loc }}+\epsilon_{t_{i+1}}^{\text {mblur }}\right)
\end{array}
$$

As shown in the Appendix, $C:=\operatorname{cov}\left(\eta_{t_{i}}, \epsilon_{t_{i+1}}^{\text {mblur }}\right) \neq 0$. The correlation relationship in Eq. 19 is used to alter the form of the standard "Corrector" or "Measurement Update" and the end result was shown in Eq. 15 above (see pgs. 116-117 in Ref. [56] for complete theoretical background). The remaining equations defining the classic KF are the same in the MBF. For example, since $\operatorname{cov}\left(\eta_{t_{i+1}}, \epsilon_{t_{i+1}}^{\mathrm{mblur}}\right)=0$ within the model considered, the "Forecast State" updates, $\hat{r}_{i+1 \mid i}=A+F \hat{r}_{i \mid i}$ and $P_{i+1 \mid i}=F P_{i \mid i} F^{\top}+Q$, associated with the classic $\mathrm{KF}$ are still valid $[\underline{55}, \underline{56]}$.

Pseudocode implementing the theoretical ideas above is provided in Algorithm 1. Note that the program flow was set up so that the classic KF could also be implemented within the $\mathrm{MBF}^{\prime}$ algorithmic framework. Eq. 15 has a fairly different form in the MBF compared to the $\overline{\mathrm{KF}}$. This change was required due to the aforementioned parallel processing of $\hat{r}_{i \mid i}$ illustrated in Fig. 2 (changing other equations from the MBF to the $\mathrm{KF}$ essentially requires changing $P_{i \mid i}$ to $P_{i+1 \mid i}$ as shown in the code provided on GitHub). One difference worth noting is that $H_{F}$ and $H_{A}$ have different definitions when Algorithm 1 is used to process the classic KF (the open-source code provided illustrates this feature). It should also be noted that although we focus on the 1D scalar case in this article, the MBF algorithm presented above is described in terms of the multivariate case.

\section{F. Illustrative Results and Introduction to Software}

To show the modeling ideas applied to practical SPT problems, we illustrate how the MBF output can process two types of trajectories commonly encountered in SPT where other analysis methods encounter problems (these trajectories exhibit some form of statistical nonstationarity). In these simulations, we generate exact realizations from the OU process shown in Eq. 1. The IPython Notebooks supplementing this work have comments describing the input parameters. We describe the basic simulation at a high-level in the next paragraph and the relevance of the results afforded by the algorithm \& software tool introduced within this article in the remaining paragraphs. Sec. III presents larger scale simulations results (studying multiple trajectories under systematically varied parameter regimes).

Before proceeding, we need to introduce some simulation parameters: $N_{\text {sub }}$ is a parameter used to model the underlying particle visiting multiple spatial locations while emitting photons used to construct a PSF in one image. To model the microscope's measurement output $\psi_{t_{i}}$, the OU trajectory was sampled at $t_{i}-\left(\frac{N_{\text {sub }}-1}{N_{\text {sub }}}\right) t_{E}, t_{i}-\left(\frac{N_{\text {sub }}-2}{N_{\text {sub }}}\right) t_{E}, \ldots, t_{i}$ and then averaged over the $N_{\text {sub }}$ samples to mimic "motion blur" in one frame. The effective localization errors induced by finite photon counts and background fluorescence on the motion blurred trajectory is modeled by a mean zero Gaussian, $\epsilon_{t_{i}}^{\text {loc }}$, and this Gaussian random variable (independent of $r$ ) is added to the discretely sampled and "blurred" trajectory. This process for simulating measurements is repeated for each of the uniformly spaced observation time. We subsequently attempted to infer/extract the parameter $\theta$ given one trajectory generated in this fashion with various estimators. IPython (Jupyter) Notebooks generating the data and graphs are provided to facilitate users implementing these techniques. Additional simulation details are deferred to these notebooks since we focus on illustrating new capabilities in this section.

The first example analyzes a trajectory where nonstationary phenomena affect the dynamics of the observed measurement sequence. In the trajectory shown in Fig. 3 , the particle is being "sucked into a harmonic well". There are large attractive forces at earlier times and as this particle relaxes into the harmonic well, these forces reduce in magnitude (the forces are precisely quantified in the bottom panel of Fig. 3). The molecule's mean position 


\begin{abstract}
Algorithm 1: Pseudocode for evaluating the innovation likelihood of common SPT models (pure, directed, and confined diffusion) given time series of correlated observations obscured by motion blur and localization measurement errors. External functions appearing below are flagged via a different font and are defined in the Supp. Mat.
\end{abstract}

1 function MotionBlurFilter
$\left(\left\{\psi_{i}\right\}_{i=1}^{T},\left\{\sigma_{\text {loc }}^{\text {Input }}(i)\right\}_{i=1}^{T}, \theta\right)$

$2 \%$ Inputs: Time series of noisy position measurements $\left\{\psi_{i}\right\}_{i=1}^{T}$, candidate parameter vector $\theta=\left(\kappa, D, \sigma_{\text {loc }}, v\right)$, and [optional: time series of localization estimates, $\left.\left\{\sigma_{\text {loc }}^{\text {Input }}(i)\right\}_{i=1}^{T}\right]$

\% Outputs: $\log$ likelihood $\log \mathcal{L}$ and filtered state series $\left\{r_{t \mid t}\right\}_{i=0}^{T}$

$3 \%$ Compute Discrete Filter Variables

${ }_{4} P_{1 \mid 0}, r_{1 \mid 0}, r_{0 \mid 0}, P_{\text {Innov }}=$ InitializeFilterPars $\left(\left\{\psi_{i}\right\}_{i=1}^{T}, \theta, \delta\right)$

$5 F, Q, A, H, Q^{\text {mblur }}, C, H_{F}, H_{A}=$

ExactMapOfContinuousToDiscrete $(\theta)$ \%Note: some auxiliary variables above are solely to allow this routine to process the classical form of the Kalman Filter.

6 filteredState $=r_{0 \mid 0} ; \log \mathcal{L}=0 \%$ Initialize variables to be returned

7 for $t=1: T$ do

$8 R_{t}=Q^{\text {mblur }}+\left(\sigma_{\text {loc }}^{\text {Input }}(t)+\sigma_{\text {loc }}\right)^{2} \%$ Compute Net

Measurement Noise Covariance at $t$

$9 \%$ Begin computation of innovation likelihood

$10 S=H P_{\text {Innov }} H^{\top}+R_{t} \quad \%$ Compute Innovation Covariance at $t$

$11 z=\sqrt{S^{-1}}\left(\psi_{t}-H_{F} r_{t-1 \mid t-1}-H_{A}\right) \quad \%$ Normalized

Innovation at $t$

$12 \log \mathcal{L}=\log \mathcal{L}+1 / 2 \log \left(\left|(2 \pi S)^{-1}\right|\right)-z^{\top} z / 2 \quad$ \%add

to $\log$ likelihood

$13 \%$ Update filter parameters for next iteration

$14 K=$ ComputeGain $\left(P_{\text {Innov }}, C, H, R_{t}, F\right)$

$15 r_{t \mid t}=r_{t \mid t-1}+K\left(\psi_{t}-H_{F} r_{t-1 \mid t-1}-H_{A}\right)$

16 filteredState.append $\left(r_{t \mid t}\right) \%$ Store filter estimate

${ }_{17} P_{t \mid t}=P_{t \mid t-1}-K\left(H P_{\text {Innov }} H^{\top}+R_{t}\right) K^{\top} \%$ Update

filter covariance

${ }_{18} P_{t+1 \mid t}=F P_{t \mid t} F^{\top}+Q \%$ Update state forecast covariance

$19 r_{t+1 \mid t}=F r_{t \mid t}+A \%$ Forecast state

$20 P_{\text {Innov }}=$ ComputeInnovCov $\left(P_{t \mid t}, P_{t+1 \mid t}\right)$

$21 \%$ Call above allows Algorithm to also process classic $\mathrm{KF}$

22 end

23 return $\log \mathcal{L}$, filteredState

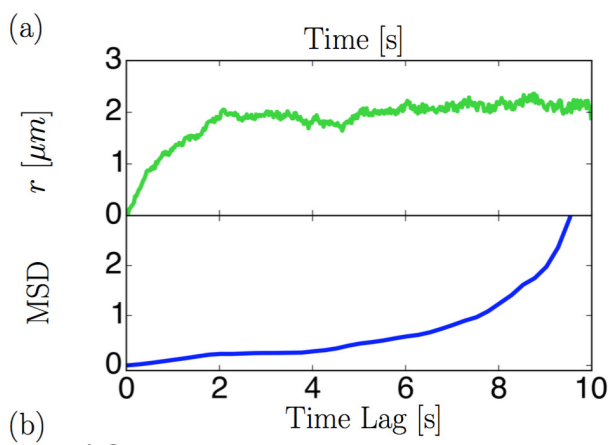

(b)

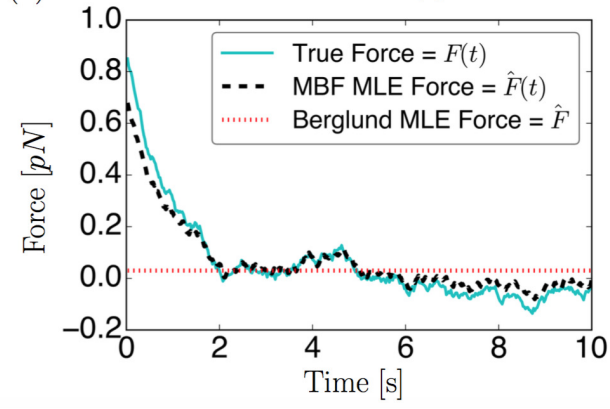

FIG. 3. Illustration of Issues Encountered by Established SPT Methods. The top panel displays the measured position of a simulated non-stationary confined trajectory. The middle panel displays the corresponding Mean Squared Displacement (MSD) vs. time lag. The bottom panel displays the true instantaneous force of the simulated particle as well as two estimates of the time dependent force (i) that of a "directed diffusion" model accounting for motion blur, but assuming a constant velocity over time and (ii) that of the Motion Blur Filter (MBF) introduced here (see text for details). Recall that position and force are quantities defined instantaneously in time for a single trajectory. This is in contrast to the information contained at single "time lag" value (denoted by $\tau)$ in the MSD vs. time lag curve. MSD curves empirically average the square of position differences $(r(t+\tau)-r(t))^{2}$ over multiple time windows where $t$ represents absolute time (the MSD curve is obtained by varying $\tau$ ). IPython Notebooks used to generate these figures are provided with this work to provide additional simulation and parameter estimation details.

and "position increments" (the latter is used in MSD) also change appreciably over time (i.e., neither the position or "confinement forces" have reached their stationary distribution [63]). This relaxation induces the primary source of statistical non-stationarity in this example. The true instantaneous velocity in these simulations can be obtained precisely via evaluating $v\left(t_{i}\right)=v-\kappa r_{t_{i}}$. The force, $F\left(t_{i}\right)$, is obtained by dividing the velocity by $\frac{D}{k_{B} T}$ at each point. We used the noisy position vs. time data, $\left\{\psi_{t_{i}}\right\}_{i=1}^{T}$, to infer the time dependent force by using $\left\{\psi_{t_{i}}\right\}_{i=1}^{T}$ and the MLE computed by the MBF algorithm.

Note that a stationarity assumption is often implicit in MSD or autocorrelation (including Fourier transform) based approaches $[\underline{38}, \underline{46}, \underline{50}$. The MSD (computed with the 400 samples) is shown in Fig. 3 and illustrates artifacts induced by the fact that the position increment distribution changes over time. The MBF's estimate of the instantaneous velocity is $\hat{v}-\hat{\kappa} r_{\hat{i} \mid i}$ (force is estimated by dividing this by $\left.\frac{\hat{D}}{k_{B} T}\right)$. Recall that, data-driven MLE pa- 
rameters are denoted by hats and $\hat{r}_{i \mid i}$ denotes the MBF's estimate (using the MLE) at the underlying position given the measurements up to time $t_{i}$ (the true position is not observable due to static and dynamic errors). It is emphasized that we do not use a finite difference (FD) scheme to estimate velocity (i.e., a FD scheme takes differences of measurements and divides by the time between observations) since realized SDE paths are not mathematically differentiable [64] Though the "drift function" (terms in front of $d t$ ) of the model can provide a mathematically well-defined "instantaneous velocity" [64]. The Supp. Mat. provides an illustration of the output of a simple FD scheme applied to this trajectory to highlight this problem. Fig. 3 also displays a modified Berglund [29] algorithm (accounting for constant velocity) estimate of average force. With data sampled at $25 \mathrm{~ms}$ for $T=400$ observations, one can obtain an accurate trajectory of both velocity and force. Note that in the parameterization shown in Eq. 1, the drift function provides a model of the instantaneous velocity (the velocity plot is shown in the Supp. Mat. Fig. 5). Extracting the instantaneous force from the drift function requires appealing to a fluctuation dissipation relationship as well as an accurate estimation of the diffusion coefficient. It is envisioned that there are many situations where one might not want a time averaged force or velocity (e.g., one would miss the "relaxation event" at earlier times of this trajectory). Furthermore, as we show in Fig. 5, the MBF allows unbiased estimation of the diffusion coefficient, $D$ over a wide range of molecular diffusivities and exposure times, whereas other state-of-the-art methods introduce biases in $D$ (and hence biases in estimated force).

The second example focuses on how our approach can utilize statistics characterizing localization uncertainty information computed in individual images. The top panel of Fig. 4 displays a confined trajectory. In this trajectory, the effective localization noise is simulated to increase over time due to photobleaching effects (introducing nonstationarity in measurement statistics). Time dependent localization noise is commonly encountered when multiple GFP dyes are used to tag a molecule and/or background, e.g. $[20,33]$. The nominal localization noise in the input sequence $\left\{\sigma_{\text {loc }}^{\text {Input }}\left(t_{i}\right)\right\}_{i=1}^{T}$ is intended to come from a typical SPT localization method applied to a PSF (e.g., $[52,53])$, however it is acknowledged that the PSF measured not only contains contributions from static and dynamic errors, but the uncertainty estimate is likely an idealized limit (hence the data-driven effective static measurement noise magnitude will differ from the input sequence). The version of the sample software provided permits estimation of a constant off-set, $\sigma_{\text {loc }}$, from the input $\left\{\sigma_{\mathrm{loc}}^{\text {Input }}\left(t_{i}\right)\right\}_{i=1}^{T}$ (the code can be easily modified to account for more complex models). If no input sequence is provided, the code estimates a constant time independent effective static measurement noise.

The (known) true static localization error magnitude of the noise added to the trajectory is shown in the bottom panel of Fig. 4. Parametric or non-parametric estimates

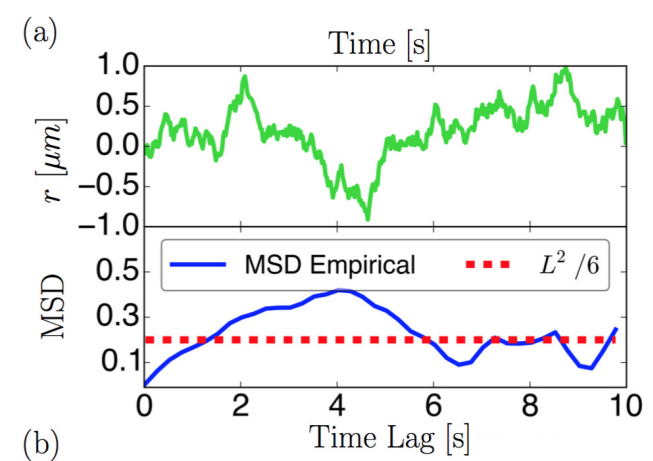

(b)

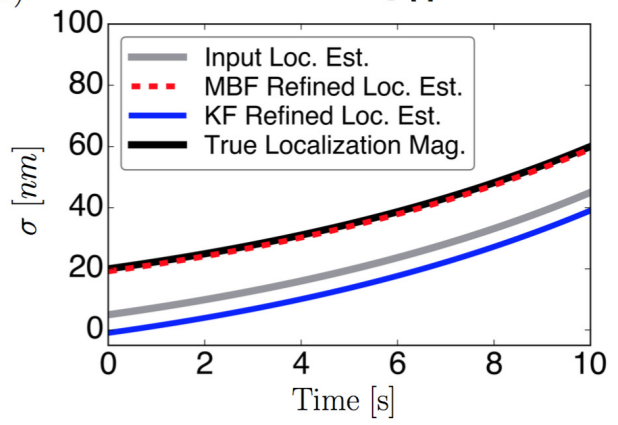

FIG. 4. Empirically Estimating a Non-stationary Effective Static Noise. The top panel displays the measured position of a simulated confined trajectory experiencing a localization noise variance changing over time. The middle panel displays the corresponding Mean Square Displacement (MSD) curve. Note the top and bottom plots have "absolute time" for the x-axis and the MSD plot uses "Time Lag" for the x-axis (see caption of Fig. 3 for additional details regarding the MSD curve). The bottom panel displays (i) the magnitude of the true time varying localization noise added to this trajectory, (ii) a nominal "Cramer-Rao" type lower bound proxy of the localization noise provided to the time series estimator (labeled as "Input Loc. Est."), (iii) two data driven-estimates of the true localization noise. One estimates uses the classic Kalman Filter (KF) and the other uses the Motion Blur Filter (MBF) to estimate the effective localization noise (note that motion blur in the measurements causes the KF to be misspecified). IPython Notebooks used to generate these figures are provided with this work to provide additional simulation and parameter estimation details.

of this type of noise trend from the time series data alone can be difficult if trajectories are not long [33] and/or if the exposure times associated with the measurements are large. Fortunately, various theoretical approximations for the lower-bound of the uncertainty associated each localization at $t_{i}$ can be obtained with established methods $[52,53,59]$. However, these uncertainty estimates often appeal to large sample Cramer-Rao bounds (CRB) $[20,53,59]$ which are not reflective of the true "static error" observed in practice. Finite sample error and real-world features (including motion blur) not accurately modeled often make the empirical data exhibit error different than the CRB. The bottom panel of Fig. 4 displays a time varying CRB type localization estimate. Even though the CRB estimates are overly optimistic, this type of time dependent localization information (i.e., uncertainty estimates provided by a 3rd party piece of 
software) can be used to aid kinetic analysis.

Both the classic KF and the MBF are able to utilize the noisily measured data and the (possibly biased) time dependent localization information afforded by image analysis [20, 53, 59] to jointly infer the kinetic parameters. Recall that the MBF code provided estimates a constant off-set adjustment $\sigma_{\text {loc }}$ to the input localization noise standard deviation (an estimated zero off-set of the localization noise implies perfect agreement with the input static noise standard deviation). The MBF can use the observations and blur information encoded in Eqs. 1- 2 to consistently estimate both the empirical effective static measurement noise and the kinetic parameters governing motion despite slightly biased time varying localization input. The KF assumes the measurements are reflective of the instantaneous position of the particle at the time of the measurement and underestimates the net static noise magnitude (this effect is expected from the results reported originally in Ref. [29] for a "pure" diffusion case).

It is emphasized throughout that accurately modeling fluctuations and measurement noise enables one to extract higher quality information from individual trajectories. For example, $L^{2} / 6$ (the long time MSD limit assuming the SDE parameters are fixed and $\kappa>0[32,45,67])$ is accurately estimated via the MBF. An MLE estimate of 0.22 was obtained from the data using a single trajectory (400 observations spaced by $25 \mathrm{~ms}$ ) without requiring the selection of tunable parameters (the true value of $L^{2} / 6$ was 0.20). In MSD computations processing a single trajectory, the "time lag" is often denoted by $\tau$ and a single point in an MSD curve fixes $\tau$ and computes the average of $(r(t+\tau)-r(t))^{2}$ over time. However, many estimates relying on the MSD include biases induced by "time lag truncation". In practice, the full trajectory is rarely used for MSD computations [20, 25] since there is less data available for larger values of $\tau$. Using a subset of the available $\tau$ values is what we refer to as "time lag truncation" error. Time lag truncation is usually carried out heuristically and the decision of where to truncate can affect parameter estimates based on MSD $[20,29]$. For example, if one averages over the last $1 / 4$ of the MSD displayed in Fig. 4, an estimate of $L^{2} / 6=$ 0.17 is obtained for the plateau value. If one ignores the first and last quarters of the data, an estimate of 0.28 is obtained (such heuristic truncations are commonly used in MSD and this is a well-known problem with MSD $[\underline{20}, \underline{29}])$. Note that MSD curves also inherently include unnecessary additional noise source (e.g., noise due to aggregating position increments over disparate times). Our approach, using the full sequence of data (without tunable parameters), is close to the truth despite the high degree of noise observed in the MSD. The ability to systematically leverage time dependent localization information (afforded by physics based models [16, $\underline{51-}$ $53,59]$ ) into the MBF and carry out likelihood inference is practical benefit of the MBF approach. Hence, we have provided examples of how to achieve this in our associated
IPython Notebooks.

\section{G. Comparison to Other Approaches}

A variety of techniques have attempted to utilize MSD approaches to quantify both static and dynamic error statistics $[38,46,50]$. However, as we illustrated in the previous subsection, MSD approaches ignore useful timeordered information. Specifically MSD methods aggregate increments from potentially disparate times. This aggregation can degrade dynamic information and complicate analyzing the MSD. Likelihood based techniques have been applied to SPT tracking problems previously $[28, \underline{34}, \underline{39}, 68]$, though, the aforementioned works ignore the time correlation effects induced by purely static error in addition to making unnecessary approximations of the likelihood function. One approximation used is the so-called Euler (sometimes referred to as the EulerMaruyama [64]) approximation. The Euler approximation is a numerical integration technique which simulates a generic SDE, $d r_{t}=\mu\left(r_{t}\right) d t+\sigma\left(r_{t}\right) d B_{t}$, via $r_{t_{i+1}}=\mu\left(r_{t_{i}}\right) \Delta t_{i}+\sigma\left(r_{t_{i}}\right) \Delta B_{t_{i}}$ where $\Delta t_{i}:=t_{i+1}-t_{i}$ and $\Delta B_{t_{i}}:=B_{t_{i+1}}-B_{t_{i}}$ (this Brownian increment can be simulated precisely, but the other numerical approximation errors can be large $[\underline{64}, \underline{69}])$. We remind the reader that Eq. 3 - 4 solve the assumed SDE and measurement equation precisely without any numerical integration or Euler type approximation (hence temporal and spatial statistics are consistent with the SDE model in Eq. 1 2). Time series methods appealing to the Euler approximation can cause a high degree of parameter estimation bias even in the measurement noise free cases $[69,70]$. Ignoring statistical time correlation induced by localization and motion blur further degrades estimates of parameters. Inaccurate likelihood approximations (like those induced by the Euler approximation) also prevent researchers from applying reliable consistency tests to fitted models since the likelihood does not correspond to the assumed model [70].

In addition to the aforementioned issues, implicit spatial or temporal stationarity assumptions are made in many SPT approaches $[\underline{34}, \underline{37}, \underline{39}, \underline{68}$. The first work (to this author's knowledge) treating static and dynamic error induced by motion blur in SPT using a likelihood based approach was Berglund's pioneering work [29]. Berglund [29] considered a constant diffusion model contaminated by static and dynamic error (extending to a "directed" or constant velocity model, where velocity is time and space independent, is straightforward due to the measurement difference formulation used as shown by the Python companion code). However, measurement difference $[\underline{29}, \underline{38}, \underline{46}, \underline{50]}$ based schemes typically make a time stationarity assumption. That is, they assume that moments and time correlations of increments of measurements, $\psi_{t+\tau}-\psi_{t}$ are independent of $t$. Stationary assumptions are also commonly made in power spectral methods [37] and "nonparametric" approaches [34]. In 
Refs. [39, 68], an approximate Bayesian approach was used to approximate temporally and spatially dependent velocity and force (an Euler approximation of the likelihood was utilized), but the approach did not account for the time correlation effects of static or dynamic measurement noise (i.e., established KF ideas were not used and the likelihood was inexact). Maximum likelihood time series estimation of parameters determining spatially dependent velocity and force using the standard KF likelihood have been studied in single-molecule manipulation studies [57, 58, 71, 72] and in SPT [33, 65]. Ref. [41] extended the KF to allow "switching linear dynamical systems" and used a nonparametric Bayesian approach to systematically determine regime switching with an exact likelihood. However, in Refs. [33, 41, 65], effects of motion blur were lumped into the effective measurement error since both $D$ and $\delta$ were small and the "exact" KF likelihood was computed corresponding to a model only technically accounting for static measurement errors.

\section{RESULTS AND DISCUSSION}

In the results that follow, we focus on analyzing $N$ time series containing $T$ observations with a uniform time spacing, $\delta$, between observations. The data is modeled as being collected in uniform continuous illumination, a common situation in cell biology [16, 29, 51]. For all simulations reported in this section, we study a constant localization noise, $\sigma_{\text {loc }}=30 n m, v=0, \kappa>0$, and initial conditions drawn from the stationary distribution in order to focus on the effects of motion blur on confined trajectories. To facilitate comparison and reduce noise due simply to random number generation, we analyze the same batch of trajectories with three estimators: the modified Berglund directed diffusion model with motion blur, the classic KF (without motion blur), and the new MBF. To illustrate that our derived variance and mean formulas are valid, we simulate $N_{\text {sub }}=100$ points spaced by $\frac{\delta}{N_{\text {sub }}}$ using the exact known solution to the OU process and average these quantities to approximate the integral in the Eq. 2. The $N_{\text {sub }}$ parameter determines the accuracy of approximating the integral in Eq. 1. We did not simulate the discrete realizations from the analytically derived motion blur results (derived in Appendix) to illustrate that our equations are correct and perform reasonably even if the uniform continuous illumination model contains discretization errors. In practice, pixelation and other factors [59] often introduce discrete sampling errors not completely captured by the motion blur model in Eq. 2 . Each parameter estimate reported in this section used $N=T=400$ and a separate MLE was obtained for each of the $N$ trajectories (i.e., trajectories were not combined to find a single parameter vector). The Supp. Mat. reports results with shorter trajectories corresponding to the main plots shown $(T=100, N=400)$.

In Fig. 5, we fix $\kappa=1 s^{-1}, v=0$, and analyze various diffusion coefficients $D=1 \times 10^{-3}, 1 \times 10^{-2}, 1 \times 10^{-1}, 0.9 \times$

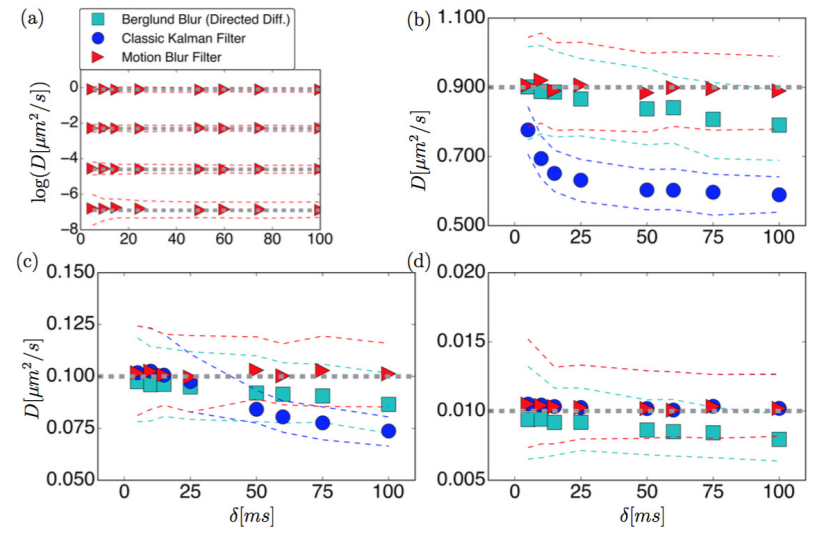

FIG. 5. Demonstration of MBF Advantages when Estimating Diffusion Coefficients. Diffusion coefficient estimation results for $\kappa=1 s^{-1}, \sigma_{\text {loc }}=30 n m, v=0$. Recall $\delta$ determines the temporal resolution of the measurement (as $\delta$ increases, both motion blur and confinement effects become pronounced). The upper left panel plots the median (symbols) and the 10th / 90th percentiles (dashed lines) of the Motion Blur Filter MLE parameter distribution computed using $N=400$ trajectories of length $T=400$ trajectories (each trajectory produced an MLE estimate) on log scale for various $D^{\prime} s$ and $\delta$ 's (each value of $D$ and $\delta$ corresponds to a summary of $N$ simulations of length $T$ ). The remaining panels zoom in on the larger $D$ cases and show results obtained by applying other estimators to the same collection of trajectories. The other likelihood based estimators fail for different reasons. Berglund's blur model [29] fails due to confinement effects becoming more pronounced as $\delta$ increases. The Kalman Filter handles confinement when motion blur effects are low (e.g., see bottom left panel), but fails when motion blur is amplified (motion blur effects increase with both $\delta$ and $D$ ). This result demonstrates the MBF is robust to a variety of regimes of relevance to SPT data modeling.

$10^{-1} \mu \mathrm{m}^{2} / \mathrm{s}$ (the latter two values were inspired by the lattice light sheet MSD results reported in Ref. [25]) and $\delta$ 's ranging from $5 \mathrm{~ms}$ to $100 \mathrm{~ms}$. In the upper left panel, we plot the median (solid symbol) and $10^{\text {th }}$ and $90^{\text {th }}$ percentiles (dashed lines) of the empirical parameter distribution obtained by analyzing the $N$ trajectories and obtaining the MLE of the MBF on logarithmic scale. The other panels zoom in on the diffusion coefficient estimates and also show the modified Berglund estimator (this estimator models motion blur and constant velocity, but ignores spatial variation in velocity) and the classic KF estimates (the KF estimator ignores motion blur, but models spatial variations in velocity). Note that the MBF consistently estimates the diffusion coefficient over the wide range of $D$ 's' and $\delta$ 's considered. The other two estimators fail for different reasons. For large $D$ and/or large $\delta$ (where large is relative to typical SPT studies), the effects of motion blur become pronounced and bias the KF's diffusion coefficient estimation. Note that for smaller $D$ (consistent with large macromolecular complexes), $\delta$ must be quite large before unmodeled motion blur affects diffusion estimation. In the Berglund estimator, the effects of nonzero $\kappa$ inducing confinement become more pronounced at larger $\delta$ and this adversely affects 
$D$ estimation regardless of the magnitude of $D$ (despite motion blur being modeled in this model). The ability of the MBF algorithm to reliably model D's relevant to SPT in the presence of varying degrees of confinement and exposure times is expected to aid researchers in SPT.
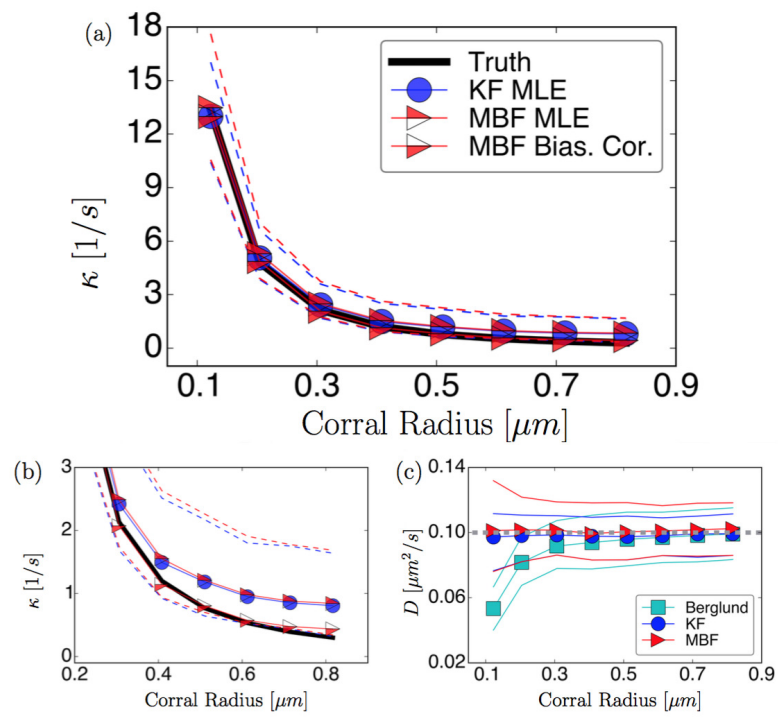

FIG. 6. Stability of the MBF in Estimating Kinetic Parameters Under Various Degrees of Confinement. Estimation of various $\kappa$ for fixed $D=0.1 \mu^{2} \mathrm{~m} / \mathrm{s}, \sigma_{\mathrm{loc}}=30 \mathrm{~nm}, \delta=25 \mathrm{~ms}, v=0$. Similar to Fig. $\underline{5}$ in terms of $N$ and $T$, except different kinetic parameters were varied. In all panels, the $x$-axis displays the so-called "corral radius" $:=\sqrt{\frac{L^{2}}{6}}$ (see Sec. II C). In the top panel, the $y$-axis displays the median $\hat{\kappa}$ (symbols) and the 10th / 90th percentiles (dashed lines) of the estimated Maximum Likelihood Estimate (MLE) parameter distribution for the corral radii explored. The bottom left panel zooms in on the "less confined" cases. $\kappa$ estimated with the Kalman Filter (KF) and Motion Blur Filter (MBF) were similar. The results of a finite $T$ bias correction [32] are also displayed (this correction is fairly close to the known true value). The bottom right panel shows the corresponding estimates of $D$ for the same trajectories and estimators considered.

Next we fix $D=0.1 \mu \mathrm{m}^{2} / \mathrm{s}$ and $\delta=25 \mathrm{~ms}$ and vary $\kappa$ in Fig. 6 (the Supp. Mat reports $\delta=10 \mathrm{~ms}$ results). This value of $D$ was selected since the classic $\mathrm{KF}$ and $\mathrm{MBF}$ were shown to be similar for this range of values. The interest is in determining the accuracy of the estimated $D$ and $\kappa$ as the latter varies in the presence of motion blur. A large positive value for $\kappa$ corresponds to high confinement or a small "corral radius". Recall that the "corral radius" $:=\sqrt{\frac{L^{2}}{6}}=\sqrt{\frac{2 D}{\kappa}}$ and the parameter $L$ quantifies the length of the region the particle can explore under confinement (see Sec. II C). At small $\delta$, estimates of $D$ are consistent with one another for both the classic $\mathrm{KF}$ and the new MBF, however the Berglund motion blur MLEs only begins to converge to the other two as the corral radius is increased (i.e., as confinement decreases). We point out that the rate of convergence of the Berglund $D$ estimate to that of the MBF (the MBF nests the Berglund estimator considered) is primarily dictated by $\delta$ for a fixed $\kappa$.
In Fig. 6, it is shown that estimates of $\kappa$ obtained using the $\overline{\mathrm{KF}}$ and $\mathrm{MBF}$ likelihoods are relatively close to one another for the corral radius values studied $(\kappa$ is not reported for the Berglund estimator since it is not included in this model). However, the median of the $\mathrm{KF}$ and MBF's MLE are biased from the known truth due to the discretely sampled finite length trajectories producing the MLE vector (the MLE mean/median converges to the truth as $T \rightarrow \infty$ for a correctly specified OU model). This finite time series sample size bias effect is known and well understood for stationary OU models sampled without measurement error [73]. If both the time series data and innovation covariance are effectively stationary and mean zero, the bias correction technique introduced in [32] for the KF (applicable to data observed with measurement error) can be heuristically applied to the MBF estimates [74]. After applying the correction outlined in Ref. [32], the bias corrected parameters of the MBF estimates are shown to coincide more closely with the true data generating process's $\kappa$. We stress that, in this example, we started in the stationary distribution (having mean zero) and the measurement noise did not vary over time. When the technical conditions hold for bias correction, the expectation of the parameter improves on average. Also note that the bias correction is derived for the expected value obtained when averaging over multiple trajectories of length $T$. The bias correction improves performance on average $[32, \underline{73}$ ], but it has a probability of degrading estimates even when all technical conditions required to apply the correction hold. If the data is confined (or is "mean reverting" [32]) around a nonzero mean at steady state, subtracting the empirical mean is a pragmatic way of "enforcing" the $v=0$ condition analyzed in Ref. [32]. Inherently non-stationary finite trajectory length bias correction requires additional research [75].

In Fig. 7, we use the innovation sequence computed at the MLE to test the quality of the model via goodness-offit tests [33, 65, 76]. When analyzing experimental live cell data, one rarely has the luxury of "ground truth", so checking modeling assumptions against data is an important step. Here, we attempt to see if the correlation induced by the (simulated) motion blur can be detected when the classic KF is applied to blurred data. For this purpose, we re-analyzed the $\delta=50 \mathrm{~ms}$ case shown in Fig. 5 and computed the $M(1,1)$ test statistic $[33,77]$. The $\bar{M}(1,1)$ test statistic aims to check if the conditional mean and correlation structure of the generalized residual series is consistent with that of a correctly specified model [77] (ignoring effects of motion blur primarily affects correlation in the generalized residual series).

We plugged in the MLE for the KF and MBF (recall that the same trajectory was fit with multiple estimators) and used the data to compute the $M(1,1)$ statistic for the $N=400$ trajectories of length $T=400$. The empirical cumulative distribution function (ECDF) of the $N=400$ test statistics is displayed for the two estimators. The vertical dashed lines plot the critical values corresponding to the limit normal null distribution of the $M(1,1)$ 
statistic [77]. The fraction of tests statistics greater than these critical values can be rejected at a nominal Type I error rate [78] indicated by the graph. By inspecting the intersection of the vertical lines with the ECDF, the fraction of the $N$ trajectories rejected for a nominal $\alpha$ can be determined. We simply picked a "conservative" and "liberal" rejection threshold to plot, however researchers can use the information encoded in the ECDF to carry out a test at any nominal Type I error level. For example, if one selected $\alpha_{\text {nominal }}=0.20, \approx 40 \%$ of the $\mathrm{KF}$ fits are rejected when motion blurred data is fit with a model not accounting for the effects of motion blur. As $T$ increases, the statistical power (ability to reject if the observed data is inconsistent with the assumed model) increases whereas the test statistics computed using the MBF innovation likelihood exhibit rejection rates just below the expected Type I error rates. To illustrate how power increases with $T$, we show results obtained using the same parameters, but increasing trajectory length to $T=1000$. Using $\alpha_{\text {nominal }}=0.20, \approx 70 \%$ of the KF fits are rejected with the increased $T$ value.

Before concluding, we make some technical notes. For likelihood based time series analysis, it is recommended that a "reasonable" number of observations are used to estimate parameters (accuracy depends on a variety of factors including $\delta, \theta, T$, etc.) Some guidance about parameter accuracy and variability in the measurement noise free case can be obtained from probability and statistical theory [73], but much theory is asymptotic in nature. Using simulations in the parameter regime of interest to quantify the bias and MLE parameter variability is recommended. The interested reader can tweak these parameters in the supplied IPython Notebooks to explore different regimes. At one extreme, if the product of $\kappa$ and $\delta$ is "large" relative to the spatial and temporal resolution afforded by the measurement device, then detecting the temporal correlations in the time series data will be problematic with finite $T$ under the model assumed in Eq. 1. In this setting, using the parameterization used here, an MLE algorithm will typically estimate the stationary variance correctly, $\frac{\hat{D}}{\hat{\kappa}}$, but the individual components may not be reflective of the underlying truth. At the other extreme, when the true $\kappa$ is near zero, other wellknown technical problems occur due to so-called "unit root" technical complications arise [55]. In SPT terms, this effectively means no appreciable confinement can be detected and the particle may be exhibiting simple "free" or "directed" diffusion. Hence if the MBF analysis predicts $\kappa \leq 0$ within statistical uncertainty, appropriate caution should be taken.

In live cell data, it is not expected that simple pure "free" or "directed" diffusion exist. Some degree of confinement (due to the inherent crowded nature of the cell) is almost always expected to be experimentally detectable in mobile particles tracked in vivo with the resolution afforded by modern optical microscopes. Despite the technical caveats stated above, we have demonstrated that high accuracy parameter estimates can be obtained for fairly wide range of $\kappa$ 's $\delta$ 's and D's relevant to SPT with reasonably "small" $T$ using the MBF.

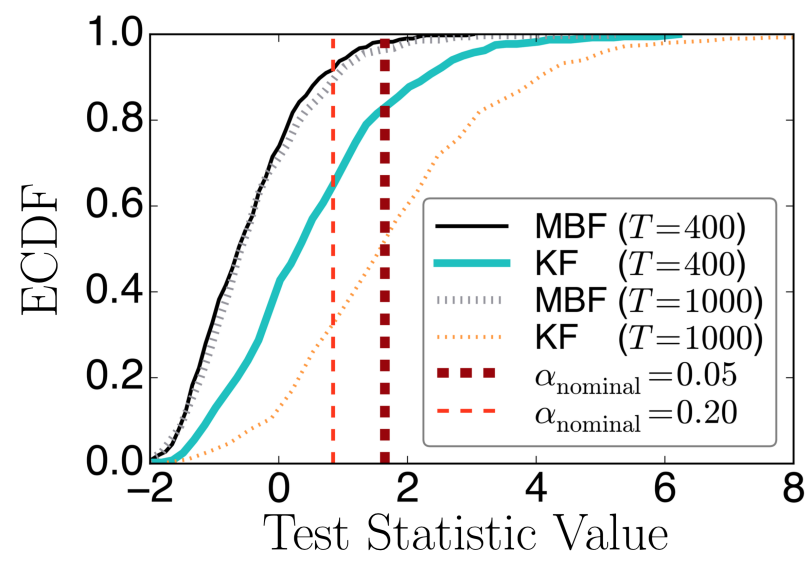

FIG. 7. Statistically Testing Model Assumptions without Ground-truth. Empirical cumulative distribution function (ECDF) obtained by evaluating the $\mathrm{M}(1,1)$ test statistic [77] at the MLE obtained $N$ using length $T$ simulations obtained with $\kappa=1 \mathrm{~s}^{-1}, D=0.1 \mu^{2} / \mathrm{s}, \sigma_{\text {loc }}=30 \mathrm{~nm}, \delta=25 \mathrm{~ms}, v=0$. Data was generated using motion blurred measurements. The MLE and the corresponding $\mathrm{M}(1,1)$ statistic of the MBF (correctly modeling motion blur, hence the "null model") and the KF (not modeling motion blur, hence representing "model misspecificaiton") were computed for each of the $N$ trajectories analyzed. The length of the trajectories $T$ was increased to show the increase in power one can obtain in this regime. Two critical values corresponding to Type I error rates $\alpha$ of the large $T$ test statistic distribution are shown as vertical lines (see text for additional details).

\section{CONCLUSIONS}

The Motion Blur Filter (MBF) algorithm was shown to be capable of consistently estimating parameters required for extracting forces and diffusion coefficients given a single trajectory contaminated by static and dynamic measurement errors. The approach can handle the three most popular SPT models (confined, directed, and "pure" diffusion). The approach can consistently estimate molecular motion parameters from individual trajectories (enabling quantification of heterogeneity) in situations where the diffusion coefficients, $D$, span four orders of magnitude and the camera exposure times range from 5-100 $\mathrm{ms}$ in the presence of confinement. As discussed in Sec. II C and elsewhere $[32, \underline{34}, \underline{39}, \underline{41}, 68]$, accurate and unbiased estimation of $D$ is important in obtaining spatially dependent effective molecular forces from position vs. time SPT data.

It was demonstrated that state-of-the-art estimators cannot consistently estimate motion parameters due either to neglected motion blur or confinement effects. Using state-of-the-art estimators which do not explicitly model confinement and motion blur results in substantial biases of $D$ (hence affecting estimates of molecular forces). Other pragmatic issues arising when analyzing individual trajectories, e.g. how to account for time varying localization 
accuracy, how to correct for parameter bias encountered when trajectories are discretely sampled with finite length samples (finite sample size bias is prevalent in time series estimation [32]), and how to test fitted models against data with the MBF were discussed and demonstrated. To facilitate implementation and promote reproducible research, we have provided Python scripts and IPython Notebooks on GitHub (this code both demonstrates general use and can also reproduce Figs. 3 - 4 ).

The approach, as presented, processed individual trajectories since a variety of microscopy techniques are now capable of producing long, high time resolution data from different imaging modalities $[\underline{1}, \underline{4}, \underline{8}, \underline{19}, \underline{20}, \underline{22}, \underline{24}-27, \underline{79}$ ]. Both the localization quality and temporal resolution can deviate substantially from theory $[\underline{50}, \underline{52}, \underline{53}, \underline{59]}$ (or even vary over time) and depend on the modality (e.g., the fluorescence channel in multicolor experiments can have different quality). Our algorithm can handle these practical complications faced by SPT researchers and produce output from different experiments which aims at removing experimental measurement and sampling artifacts to produce motion parameters representative of the true underlying tagged particle. As we demonstrated in this work, a large source of bias introduced by the experimental apparatus is "motion blur". The software provided can be used to empirically explore different regimes of interest and the accuracy afforded by the MBF approach. If a researcher desires to estimate one of the three popular SPT motion models (confined/corralled, directed, "pure" diffusion, or some combination of these), it is recommended to use the MBF since the algorithm has demonstrated accuracy in many regimes of relevance to SPT (whereas other estimators are biased in some regimes).

If it is discovered or believed that the parameters characterizing the dynamics driving the motion of the molecule(s) of interest at a given spatial location in a cell are independent of time $[\underline{4}, \underline{34}, \underline{39}]$, one can modify our algorithm to aggregate multiple time series even if they have vastly different localization precisions or exposure times. For example, one could use each trajectory to produce a likelihood function (given trajectory specific localization information) and then develop a cost function which aims to find the single parameter vector minimizing the net sum of the log likelihoods. However, this trajectory aggregation requires a strong assumption regarding spatial and temporal stationarity and we believe that potentially interesting transient molecular events will be missed by this type of approach [33, 41], hence we advise researchers to start by analyzing data on a trajectory-wise basis $[58,71]$. In addition to parameter estimation, the likelihood based scheme provides diagnostic statistics which can be used to check statistical modeling assumptions directly against data via goodness-of-fit tests without "ground truth" (checking both shape and/or statistical dependence assumptions implicit in the model $[33,65,76])$. We demonstrated the ability of these tests to detect unmodeled correlations in the classic KF induced by motion blur effects. However, the same hypothesis testing procedure can also be used to determine whether the assumptions required to carry out the "trajectory aggregation" mentioned above (e.g., use many different trajectories to estimate a parameter vector characterizing the dynamics at a fixed spatial location) are justified by the empirical data.

The MBF estimator leveraged signal processing and stochastic process ideas to synthesize a new algorithm capable of addressing many open practical issues facing SPT data analysis. The dynamical model underlying the MBF is a continuous time linear SDEs driven by "standard Brownian diffusion" [64]. As stated in the Introduction, "anomalous diffusion" can result when one averages over many types of dynamical states [43, 44], however resolution afforded by contemporary microscopes permits temporal resolution where standard diffusion models are useful. A primary aim of this work is to provide a computational tool which can be leveraged when the molecular events of interest occur within the spatial and temporal resolution of optical microscopes before signatures of "anomalous diffusion" manifest themselves in the data. Under these conditions, backing out "effective forces" from the local molecular diffusivity is reasonable [33, 41, 66]. For longer trajectories, this may require one to segment trajectories into distinct kinetic states [41] and then apply the analysis to the segments. We did not present segmentation results, but the likelihood based MBF algorithm can be used to modify the cost function of existing stateof-the-art time series segmentation algorithms [41, 80] and remove artifacts induced by motion blur and unknown localization noise (these noise sources are ubiquitous in SPT data analysis).

Explicitly accounting for motion blur is also expected to facilitate segmenting data where multiple imaging modalities (where data is acquired with different temporal resolutions and/or exposure times) are combined to describe the dynamics of individual molecules in living cells. We presented 1D (scalar) illustrative examples, but the MBF algorithm can process multivariate signals. However, obtaining closed-form expressions for the filter quantities is slightly complicated by matrix exponentials. The computational challenges with multivariate extensions is left to future work.

\section{ACKNOWLEDGMENTS}

The author thanks Kerry Bloom for helpful motivating discussions and comments related to this as well as Kerry Bloom, Scott Lundberg, and two anonymous referees who provided valuable comments which improved the exposition of this paper. This research was supported by internal R\&D funds of Ursa Analytics, Inc. 


\section{APPENDIX}

The well-known solution to the Ornstein-Uhlenbeck (OU) SDE (Eq. 1) can be written explicitly as:

$$
r_{\delta}=A+F r_{0}+\int_{0}^{\delta} \exp (\kappa(s-\delta)) \sqrt{2 D} d B_{s},
$$

where $A \equiv(1-\exp (-\delta \kappa)) \frac{v}{\kappa}$ and $F \equiv \exp (-\delta \kappa)[64]$. The solution above can be written in terms of simple Gaussian random variables since the OU process is one of the rare cases where a SDE can be solved in closed-form by appealing to integration factor techniques used in standard ordinary differential equations. "Solved" means the process can be written explicitly in terms of time and a Brownian motion path. When an SDE is "solved", realizations can be constructed without numerical integration approximations of any deterministic or stochastic integrals [64]. The expression above can be used to compute closed-form expressions for:

$$
\begin{aligned}
\mathbb{E}\left[r_{\delta} \mid r_{0}\right] & =A+F r_{0} \\
\mathbb{E}\left[\frac{1}{\delta} \int_{0}^{\delta} r_{\delta} \mid r_{0}\right]= & H_{A}+H_{F} r_{0} \\
H_{F}= & \frac{1}{\delta} \int_{0}^{\delta} \exp (-s \kappa) d s \\
& =\frac{1}{\kappa \delta}(1-\exp (-\delta \kappa)) \\
H_{A}= & \frac{1}{\delta} \int_{0}^{\delta}(1-\exp (-s \kappa)) \mu d s \\
& =\mu-\frac{\mu}{\delta \kappa}+\frac{\mu \exp (-\delta \kappa)}{\delta \kappa} \\
\mu:= & \frac{v}{\kappa}
\end{aligned}
$$

where $\delta>0$ (note all discrete parameters defined in this Appendix depend implicitly on $\delta$ ).

Since the OU process considered is linear and driven by standard Brownian motion, it is characterized by the first two moments and covariances of the process. The means have been defined above. The second moments and covariances can also be computed as an explicit function of time and $\theta$ for the model considered. Recall that the KF and MBF uncertainty estimates both are centered around using covariances of mean zero estimates to make various linear projections [56]. In what follows, without loss of generality, we assume $r_{0}$ is statistically independent of $B_{t} \forall t>0$ and that $r_{0}=0, v=0$ (so the relation $\operatorname{cov}\left(r_{t}, r_{s}\right)=\mathbb{E}\left[r_{t} r_{s}\right]$ holds for $s \leq t$ ), and $\kappa \geq 0$. Under these non-restrictive conditions and using the well-known quadratic variation properties of Brownian motion [64], one has the following closed-form relationship for the state covariance [64]:

$$
\begin{aligned}
\operatorname{cov}\left(r_{t}, r_{s}\right) & := \\
& \mathbb{E}\left[\int_{0}^{t} \exp (\kappa(u-t)) \sqrt{2 D} d B_{u} \int_{0}^{s} \exp (\kappa(v-s)) \sqrt{2 D} d B_{v}\right] \\
& =2 D \int_{0}^{s} \exp (\kappa(v-t)) \exp (\kappa(v-s)) d v \\
& =\frac{D}{\kappa}(\exp (2 \kappa s)-1) \exp (-\kappa(s+t))
\end{aligned}
$$

The above expression is valid for $s \leq t$. The state covariance above can be used to solve for the variance and covariance of other quantities required by the MBF. Specifically one needs to compute both $\mathbb{E}\left[\frac{r_{\delta}}{\delta} \int_{0}^{\delta} r_{t} d t\right]$ and $\mathbb{E}\left[\frac{1}{\delta} \int_{0}^{\delta} r_{t} d t \times \frac{1}{\delta} \int_{0}^{\delta} r_{s} d s\right]$ to solve the "Corrector" update (see Fig. 2). The former expectation provides $\operatorname{cov}\left(r_{i}, \tilde{\psi}_{i}\right)$ and the latter provides the contribution of motion blur, $\operatorname{cov}\left(\epsilon_{t_{i}}^{\mathrm{mblur}}, \epsilon_{t_{i}}^{\mathrm{mblur}}\right)$, to the net measurement covariance $\operatorname{cov}\left(\tilde{\psi}_{i}, \tilde{\psi}_{i}\right)$ stated in Eq. 12. Note that in the MBF where uniform illumination is assumed, we use both $\delta$ and $t_{E}$ to represent the exposure time. The order of time integration and expectation can be exchanged for $\delta>0$ for the process considered, e.g. $\mathbb{E}\left[\frac{r_{\delta}}{\delta} \int_{0}^{\delta} r_{t} d t\right]=\frac{1}{\delta} \int_{0}^{\delta} \mathbb{E}\left[r_{\delta} r_{t}\right] d t[\underline{64}]$. This reduces the problem to solving standard time integrals, since $\mathbb{E}\left[r_{t} r_{s}\right]=\operatorname{cov}\left(r_{t}, r_{s}\right)$ (recall our non-restrictive assumptions on the process mean) has already been solved in terms of $\theta, t$ and $s$ as shown above. With this background, it can also be shown that:

$$
\begin{aligned}
\delta & =t_{i}-t_{i-1}=t_{E} \forall i \\
Q^{\text {mblur }} & :=\operatorname{cov}\left(\epsilon_{t_{i}}^{\text {mblur }}, \epsilon_{t_{i}}^{\text {mblur }}\right) \\
& =\frac{D}{\kappa \delta^{2}}\left(\frac{2 \delta}{\kappa}-\frac{3}{\kappa^{2}}+\frac{4}{\kappa^{2} e^{\delta \kappa}}-\frac{1}{\kappa^{2} e^{2 \delta \kappa}}\right) \\
C & =\operatorname{cov}\left(\eta_{t_{i-1}}, \epsilon_{t_{i}}^{\text {mblur }}\right) \\
& =\frac{D}{\kappa \delta}\left(\frac{1}{\kappa}-\frac{2 \exp (-\kappa \delta)}{\kappa}+\frac{\exp (-2 \kappa \delta)}{\kappa}\right)
\end{aligned}
$$

The first line in the set of equations above is to remind the reader that it is assumed no "missing frames" exist in the uniform illumination measurement model stated in Eq. 2 . Missing frames may occur in time lapse experiments or if quantum dots blink (both missing frames and more exotic shutter functions could be considered within the MBF framework, but these cases require a more complicated notation which we have elected not to explore in this article which introduces the basic MBF). Combining the expressions for $\left(Q^{\text {mblur }}, C\right)$ above, with the expressions for $(A, F)$ reported previously (see below Eq. 20), $Q=$ $\operatorname{cov}\left(r_{\delta}, r_{\delta}\right)$ (see Eq. 27) and the expressions for $\left.\overline{(H}_{F}, H_{A}\right)$ (see Eq. 22) provide the closed-form expressions required to precisely discretize the model reported in Eqs. 1 and $\underline{2}$ without any statistical approximations in the filtering framework. We remind the reader that all the quantities derived depend implicitly on both $\delta$ and $\theta$. 
[1] N. Arhel, A. Genovesio, K. Kim, S. Miko, E. Perret, J. Olivo-Marin, S. Shorte, and P. Charneau, Nature Methods 3, 817 (2006).

[2] B. Brandenburg and X. Zhuang, Nature Reviews. Microbiology 5, 197 (2007).

[3] S. Lange, Y. Katayama, M. Schmid, O. Burkacky, C. Bräuchle, D. C. Lamb, and R.-P. Jansen, Traffic (Copenhagen, Denmark) 9, 1256 (2008).

[4] S. Manley, J. Gillette, G. Patterson, H. Shroff, H. Hess, E. Betzig, and J. Lippincott-Schwartz, Nature Methods 5, 155 (2008).

[5] C. Joo, H. Balci, Y. Ishitsuka, C. Buranachai, and T. Ha, Annual review of biochemistry 77, 51 (2008).

[6] J. Biteen, Biopolymers 95, 287 (2011).

[7] G. Danuser, Cell 147, 973 (2011).

[8] S. Ram, D. Kim, R. J. Ober, and E. S. Ward, Biophysical journal 103, 1594 (2012).

[9] E. Meijering, O. Dzyubachyk, and I. Smal, Methods in enzymology 504, 183 (2012).

[10] E. de Wit and W. de Laat, Genes \& development 26, 11 (2012).

[11] N. Pawar, C. Donth, and M. Weiss, Current biology : CB 24, 1905 (2014).

[12] S. E. Polo and S. P. Jackson, Genes \& development 25, 409 (2011).

[13] R. Rohatgi, L. Milenkovic, and M. P. Scott, Science (New York, N.Y.) 317, 372 (2007).

[14] H. Hajjoul, J. Mathon, H. Ranchon, I. Goiffon, J. Mozziconacci, B. Albert, P. Carrivain, J.-M. Victor, O. Gadal, K. Bystricky, and A. Bancaud, Genome research 23, 1829 (2013).

[15] F. Ye, D. K. Breslow, E. F. Koslover, A. J. Spakowitz, W. J. Nelson, and M. V. Nachury, eLife 2, e00654 (2013).

[16] A. Gahlmann and W. E. Moerner, Nature Reviews Microbiology 12, 9 (2014).

[17] V. Levi, Q. Ruan, M. Plutz, A. S. Belmont, and E. Gratton, Biophysical journal 89, 4275 (2005).

[18] S. R. P. Pavani, M. A. Thompson, J. S. Biteen, S. J. Lord, N. Liu, R. J. Twieg, R. Piestun, and W. E. Moerner, Proceedings of the National Academy of Sciences of the United States of America 106, 2995 (2009).

[19] N. P. Wells, G. A. Lessard, P. M. Goodwin, M. E. Phipps, P. J. Cutler, D. S. Lidke, B. S. Wilson, and J. H. Werner, Nano letters 10, 4732 (2010).

[20] M. A. Thompson, J. M. Casolari, M. Badieirostami, P. O. Brown, and W. E. Moerner, Proceedings of the National Academy of Sciences of the United States of America 107, 17864 (2010).

[21] T. T. Ashley, C. Chan-Tse, and S. B. Andersson, Proceedings of the 2012 IEEE Conference on Decision and Control , 2328 (2012).

[22] M. P. Backlund, R. Joyner, K. Weis, and W. E. Moerner, Molecular Biology of the Cell 25, 3619 (2014).

[23] P. A. Vasquez and K. Bloom, Nucleus 5, 376 (2014).

[24] K. Welsher and H. Yang, Nature nanotechnology 9, 198 (2014).

[25] B.-C. Chen, W. R. Legant, K. Wang, L. Shao, D. E. Milkie, M. W. Davidson, C. Janetopoulos, X. S. Wu, J. A. Hammer, Z. Liu, B. P. English, Y. Mimori-Kiyosue, D. P. Romero, A. T. Ritter, J. Lippincott-Schwartz, L. FritzLaylin, R. D. Mullins, D. M. Mitchell, J. N. Bembenek,
A.-C. Reymann, R. Bohme, S. W. Grill, J. T. Wang, G. Seydoux, U. S. Tulu, D. P. Kiehart, and E. Betzig, Science 346, 1257998 (2014).

[26] D. Li, L. Shao, B.-C. Chen, X. Zhang, M. Zhang, B. Moses, D. E. Milkie, J. R. Beach, J. a. Hammer, M. Pasham, T. Kirchhausen, M. a. Baird, M. W. Davidson, P. Xu, and E. Betzig, Science 349, aab3500 (2015).

[27] F. Wu, E. Van Rijn, B. G. C. Van Schie, J. E. Keymer, and C. Dekker, Frontiers in Microbiology 6, 1 (2015).

[28] D. Montiel, H. Cang, and H. Yang, J Phys Chem B 110, 19763 (2006).

[29] A. J. Berglund, Physical Review. E 82, 011917 (2010).

[30] S. C. Weber, M. A. Thompson, W. E. Moerner, A. J. Spakowitz, and J. A. Theriot, Biophysical journal 102, 2443 (2012).

[31] F. Persson, M. Lindén, C. Unoson, and J. Elf, Nature methods 10, 265 (2013).

[32] C. P. Calderon, Phys. Rev. E 88, 012707 (2013).

[33] C. P. Calderon, M. A. Thompson, J. M. Casolari, R. C. Paffenroth, and W. E. Moerner, J Phys Chem B 117, 15701 (2013).

[34] N. Hoze and D. Holcman, Biophys. J. 107, 3008 (2014).

[35] C. P. Calderon, Molecules 19, 18381 (2014).

[36] J.-W. van de Meent, J. E. Bronson, C. H. Wiggins, and R. L. Gonzalez, Biophysical journal 106, 1327 (2014).

[37] C. L. Vestergaard, P. C. Blainey, and H. Flyvbjerg, Physical Review E - Statistical, Nonlinear, and Soft Matter Physics 89, 022726 (2014).

[38] M. P. Backlund, R. Joyner, and W. E. Moerner, Physical Review E 91, 062716 (2015).

[39] M. E. Beheiry, M. Dahan, and J.-B. Masson, Nature Methods 12, 594 (2015).

[40] K. E. Hines, J. R. Bankston, and R. W. Aldrich, Biophysical Journal 108, 540 (2015).

[41] C. P. Calderon and K. Bloom, PloS ONE 10, e0137633 (2015).

[42] N. Monnier, Z. Barry, H. Y. Park, K.-C. Su, Z. Katz, B. P. English, A. Dey, K. Pan, I. M. Cheeseman, R. H. Singer, and M. Bathe, Nature methods 12, 838 (2015).

[43] F. Höfling and T. Franosch, Reports on Progress in Physics. Physical Society (Great Britain) 76, 046602 (2013).

[44] R. Metzler, J.-H. Jeon, A. G. Cherstvy, and E. Barkai, Physical chemistry chemical physics : PCCP 16, 24128 (2014).

[45] A. Kusumi, Y. Sako, and M. Yamamoto, Biophysical Journal 65, 2021 (1993).

[46] N. Destainville and L. Salomé, Biophysical journal 90 , L17 (2006).

[47] M. J. Saxton, Biophysical Journal 72, 1744 (1997).

[48] K. Chen, B. Wang, J. Guan, and S. Granick, ACS Nano 7, 8634 (2013).

[49] A. V. Weigel, M. M. Tamkun, and D. Krapf, Proceedings of the National Academy of Sciences of the United States of America 110, E4591 (2013).

[50] T. Savin and P. S. Doyle, Biophysical Journal 88, 623 (2005).

[51] Z. Liu, L. Lavis, and E. Betzig, Molecular Cell 58, 644 (2015).

[52] R. E. Thompson, D. R. Larson, and W. W. Webb, Biophysical Journal 82, 2775 (2002). 
[53] R. J. Ober, S. Ram, and E. S. Ward, Biophysical J. 86 , 1185 (2004).

[54] T. T. Ashley and S. B. Andersson, Physical review. E, Statistical, nonlinear, and soft matter physics 92, 052707 (2015).

[55] J. Hamilton, Time Series Analysis (Princeton University Press, Princeton, NJ, 1994).

[56] B. Anderson and J. Moore, Optimal Filtering (PrenticeHall, Englewood Cliffs, NJ, 1979).

[57] C. P. Calderon, N. Harris, C. Kiang, and D. Cox, J. Mol. Recognit. 22, 356 (2009).

[58] C. P. Calderon, W. Chen, N. Harris, K. Lin, and C. Kiang, J. Phys.: Condens. Matter 21, 034114 (2009).

[59] A. V. Abraham, S. Ram, J. Chao, E. S. Ward, and R. J. Ober, Proc SPIE Int Soc Opt Eng 7570, 757004 (2010).

[60] See Supplemental Material at [URL will be inserted by publisher] for additional results and algorithmic details.

[61] This subscript notation is standard in SDE modeling [64].

[62] The MBF was introduced in a form motivating by continuous illumination [16, 29]. The basic approach MBF can be modified to handle other "shutter functions" [29] enabling camera modes outside of continuous illumination, we focus on continuous uniform illumination since this mode is most commonly used in biophysics and cell biology experiments $[16, \underline{29}, \underline{51}$.

[63] H. Risken, The Fokker-Planck Equation (Springer-Verlag, 1996).

[64] P. Kloeden and E. Platen, Numerical Solution of Stochastic Differential Equations (Springer-Verlag, Berlin, 1992).

[65] C. P. Calderon, L. E. Weiss, and W. E. Moerner, Phys. Rev. E 89, 052705 (2014).

[66] D. Holcman, N. Hoze, and Z. Schuss, Biophysical Journal 109, 1761 (2015).

[67] H. Y. Park, A. R. Buxbaum, and R. H. Singer, Methods in enzymology (chapter 18) 472, 387 (2010).

[68] G. Voisinne, A. Alexandrou, and J.-B. Masson, Biophysical Journal 98, 596 (2010).

[69] B. Jensen and R. Poulsen, Journal of Derivatives 9, 18 (2002).

[70] C. P. Calderon, Multiscale Model. Simul. 6, 656 (2007).

[71] C. P. Calderon, N. Harris, C. Kiang, and D. Cox, J. Phys. Chem. B 113, 138 (2009).

[72] V. I. Fernandez, P. Kosuri, V. Parot, and J. M. Fernandez, Rev Sci Instrum 80, 113104 (2009).

[73] C. Y. Tang and S. X. Chen, Journal of Econometrics 149, 65 (2009).

[74] Note that the MBF has a time integrated measurement (this feature was not considered in Ref. [32]), hence the bias correction outlined in Ref. [32] may require modification in certain very high motion blur regimes (however, in the parameter regimes explored in this work, the bias correction for $\kappa$ was accurate).

[75] A general heuristic of potential pragmatic value in SPT should be noted regarding this statement: if particles are approaching their steady state distribution from "nonequilibrium conditions", bias in the estimated $\kappa$ tends to be small since there is more "relaxation" information encoded in the time series sequence and the practical utility of a bias correction scheme is reduced as can be verified empirically using the code provided.

[76] C. P. Calderon, J Phys Chem B 114, 3242 (2010).

[77] Y. Hong and H. Li, Rev. Fin. Studies 18, 37 (2005).

[78] "Nominal" is used since the normal distribution requires $T \rightarrow \infty$ for these to be the true Type I error rates $\alpha$ [77].

[79] P. Moens, M. Digman, and E. Gratton, Biophysical Journal 108, 1448 (2015).

[80] E. Fox, E. B. Sudderth, M. I. Jordan, and A. S. Willsky, IEEE Transactions on Signal Processing 59, 1569 (2011). 